\title{
A vaccine targeting the RBD of the S protein of SARS-CoV-2 induces protective immunity
}

https://doi.org/10.1038/s41586-020-2599-8

Received: 17 March 2020

Accepted: 23 July 2020

Published online: 29 July 2020

Check for updates

\section{Jingyun Yang ${ }^{1,4}$, Wei Wang ${ }^{1,14}$, Zimin Chen ${ }^{1,14}$, Shuaiyao Lu $^{2,14}$, Fanli Yang ${ }^{1,4}$, Zhenfei $\mathrm{Bi}^{1,14}$, Linlin Bao ${ }^{3,14}$, Fei Mo', Xue Li', Yong Huang', Weiqi Hong', Yun Yang' ', Yuan Zhao', Fei Ye', Sheng Lin', Wei Deng ${ }^{3}$, Hua Chen', Hong Lei', Ziqi Zhang', Min Luo', Hong Gao ${ }^{3}$, Yue Zheng', Yanqiu Gong', Xiaohua Jiang', Yanfeng $\mathrm{Xu}^{3}$, Qi $\mathrm{Lv}^{3}$, Dan $\mathrm{Li}^{1}$, Manni Wang', Fengdi Li ${ }^{3}$, Shunyi Wang ${ }^{3}$, Guanpeng Wang ${ }^{3}$, Pin $\mathrm{Yu}^{3}$, Yajin $\mathbf{Q u}^{3}$, Li Yang', Hongxin Deng', Aiping Tong', Jiong Li', Zhenling Wang', Jinliang Yang', Guobo Shen', Zhiwei Zhao', Yuhua Li', Jingwen Luo', Hongqi Liư ', Wenhai $\mathrm{Yu}^{2}$, Mengli Yang ${ }^{2}$, Jingwen $\mathrm{Xu}^{2}$, Junbin $\mathrm{Wang}^{2}$, Haiyan $\mathrm{L}^{2}$, Haixuan Wang ${ }^{2}$, Dexuan Kuang' ${ }^{2}$ Panpan Lin', Zhengtao Hu', Wei Guo ${ }^{5}$, Wei Cheng', Yanlin $\mathrm{He}^{5}$, Xiangrong Song', Chong Chen', Zhihong Xue', Shaohua Yao', Lu Chen', Xuelei Ma', Siyuan Chen', Maling Gou', Weijin Huang ${ }^{4}$, Youchun Wang ${ }^{4}$, Changfa Fan ${ }^{4}$, Zhixin Tian ${ }^{6}$, Ming Shi ${ }^{7}$, Fu-Sheng Wang', Lunzhi Dai', Min Wu', Gen Li', Guangyu Wang ${ }^{8}$, Yong Peng', Zhiyong Qian', Canhua Huang', Johnson Yiu-Nam Lau ${ }^{10}$, Zhenglin Yang" ${ }^{11}$, Yuquan Wei', Xiaobo Cen ${ }^{1,5}$, Xiaozhong Peng ${ }^{2,12}$, Chuan Qin ${ }^{3}$, Kang Zhang ${ }^{8 凶}$, Guangwen $\mathrm{Lu}^{1,13 凶}$ \& Xiawei Wei ${ }^{1 \bowtie}$}

Severe acute respiratory syndrome coronavirus 2 (SARS-CoV-2) causes a respiratory disease called coronavirus disease 2019 (COVID-19), the spread of which has led to a pandemic. An effective preventive vaccine against this virus is urgently needed. As an essential step during infection, SARS-CoV-2 uses the receptor-binding domain (RBD) of the spike protein to engage with the receptor angiotensin-converting enzyme 2 (ACE2) on host cells ${ }^{1,2}$. Here we show that a recombinant vaccine that comprises residues 319-545 of the RBD of the spike protein induces a potent functional antibody response in immunized mice, rabbits and non-human primates (Macaca mulatta) as early as 7 or 14 days after the injection of a single vaccine dose. The sera from the immunized animals blocked the binding of the RBD to ACE2, which is expressed on the cell surface, and neutralized infection with a SARS-CoV-2 pseudovirus and live SARS-CoV-2 in vitro. Notably, vaccination also provided protection in non-human primates to an in vivo challenge with SARS-CoV-2. We found increased levels of RBD-specific antibodies in the sera of patients with COVID-19. We show that several immune pathways and CD4 T lymphocytes are involved in the induction of the vaccine antibody response. Our findings highlight the importance of the RBD domain in the design of SARS-CoV-2 vaccines and provide a rationale for the development of a protective vaccine through the induction of antibodies against the RBD domain.
SARS-CoV-2, which causes a respiratory disease known as COVID-19, is responsible for a pandemic declared by the World Health Organization. Person-to-person transmission has been documented ${ }^{3,4}$. There is an urgent need for an effective preventive vaccine.SARS-CoV-2 binds to cells using human ACE2 as a receptor ${ }^{1,2}$. On the basis of our knowledge of the viral envelope protein, we hypothesized that the RBD could be a good immunogen and that it could trigger a potent functional antibody response that could neutralizeSARS-CoV-2 in vitro and in vivo in an SARS-CoV-2 infection model of non-human primates (M. mulatta) by blocking the binding of the viral envelope protein to its host cell receptor ACE2.

The baculovirus expression system was chosen to express the various proteins used in our study as this is a commercially feasible system and can manufacture the candidate vaccine-if successful-at a commercial scale, and the vaccines generated using this system are generally folded in the correct conformation of the protein ${ }^{5,6}$. In fact, this technology was used in several commonly used vaccine products, including some of the vaccines against human papilloma virus, which causes cervical cancer, and vaccines against influenza that are currently in use in Europe and the USA ${ }^{5,6}$. We aimed to assess the potential of a candidate vaccine based on the RBD domain of SARS-CoV-2, to evaluate the appropriate dosing regimen, to test its effect in generating neutralizing activity against SARS-CoV-2 in recipient animals and to determine the immune pathways involved in the generation of the immune response, to provide the groundwork for the design of an effective SARS-CoV-2 preventive vaccine.

\section{Characterization of the recombinant RBD protein}

The recombinant RBD of the spike protein of SARS-CoV-2 was prepared using insect cells and the Bac-to-Bac baculovirus expression system 


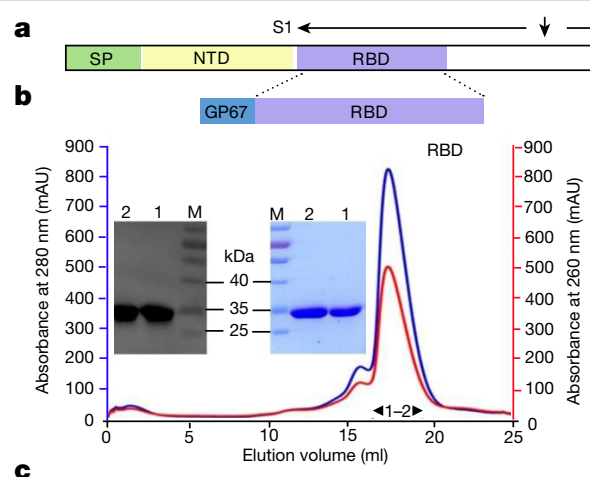

c

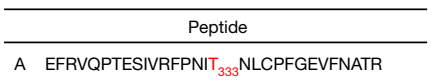

$$
\begin{aligned}
& \begin{array}{llr}
\text { O-Glycosylation } & \text { B } & \text { LPDDFTGCVIAWNS }_{438} \text { NNLDS }_{443} \mathrm{~K} \\
& \text { C } & \text { VVVLS }_{514} \text { FELLHAPAT }_{523} \text { VCGPK }
\end{array}
\end{aligned}
$$

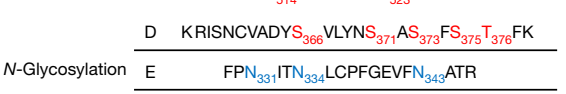

d

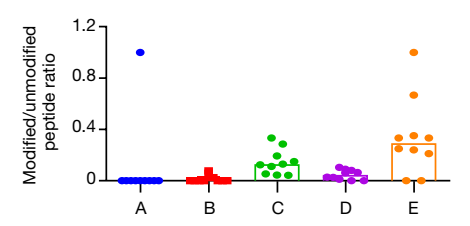

Fig. 1 Characterization of the RBD domain of the spike protein of SARS-CoV-2. a, A schematic view of the SARS-CoV-2 spike protein. The indicated domains and elements, including the signal peptide (SP), N-terminal domain (NTD), RBD, heptad repeats 1 and 2 (HR1 and HR2), transmembrane domain (TM) and cytoplasmic domain (CP), are marked. For the preparation of the RBD of the spike protein, the RBD region was engineered to include an N-terminal GP67 signal peptide. $\mathbf{b}$, A representative elution chromatograph of the recombinant RBD protein using a Superdex 200 increase column. The insets show the SDS-PAGE and western-blotting analyses of the eluted RBD samples. $m A U$, milli-absorbance units; M, marker; 1 , the eluted sample of the ascending part of the protein peak; 2 , the eluted sample of the descending part of the peak. c, Glycosylated peptides in the RBD identified by mass spectrometry. The $\mathrm{N}$-glycosylation (peptide $\mathrm{E}$ ) and $\mathrm{O}$-glycosylation (peptides $\mathrm{A}-\mathrm{D}$ ) sites are marked with residue numbers. $\mathbf{d}$, The abundance of glycosylation. The number

as previously described ${ }^{7,8}$. A GP67 signal peptide sequence was added to ensure protein secretion (Fig. 1a). Recombinant RBD was successfully obtained from the cell culture supernatant and purified to high homogeneity. The purity of the final protein preparation was more than $98 \%$ (Fig. 1b).

The apparent molecular mass of the purified RBD protein was determined to be around $34 \mathrm{kDa}$, which was approximately a quarter larger than the molecular mass calculated using the RBD amino acid sequence alone (about $27 \mathrm{kDa}$ ), suggesting that the RBD is densely glycosylated. Using mass spectrometry (MS), the intact N-glycopeptides and glycans were analysed with the GPSeeker software ${ }^{8,9}$. Three $\mathrm{N}$-glycosylation sites on asparagine were identified (Fig. 1c). O-Glycosylation sites were also evaluated by analysing the MS results using SEQUEST in Proteome Discoverer (version 2.3). Some well-known O-linked glycans such as HexNAc and Hex(1)HexNAc(1) were searched as potential variable modifications ${ }^{9,10}$. In total, ten O-glycosylation sites were identified (Fig. 1c). To determine the abundance of glycosylation, the number of MS/MS spectra of glycosylated peptides and their corresponding unmodified peptides were determined. A much higher degree of $\mathrm{N}$-glycosylation than O-glycosylation was observed (Fig. 1d).

These identified glycosylation sites were further mapped on the complex structure of the RBD of SARS-CoV-2 bound to ACE2. Most of the sites were located in the RBD core subdomain (Fig. 1e). In addition,

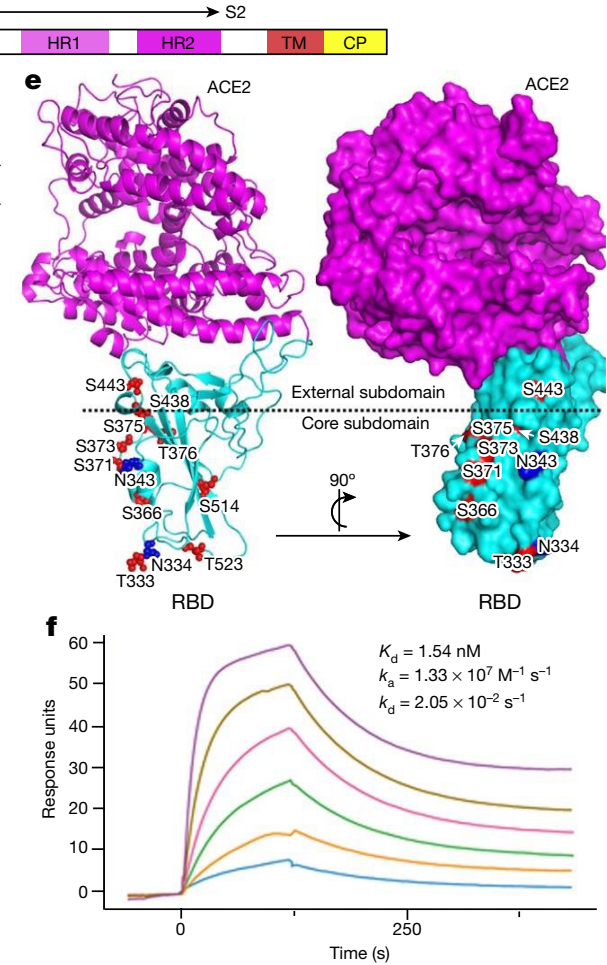

of MS/MS spectra of each glycosylated peptide listed in c and the corresponding unmodified peptides were counted. Ten MS runs were performed in which different methods for protein precipitation, models of mass spectrometry and LC-MS/MS parameters were used. Each dot shows the results of one run. e, An overview of the glycosylation sites illustrated on the basis of the solved complex structure of the RBD of SARS-CoV-2 bound to ACE2 (Protein Data Bank (PDB) code: 6LZG). The identified sites, coloured red for $\mathrm{O}$-glycosylation and blue for $\mathrm{N}$-glycosylation, are shown as spheres and are labelled. The approximate boundary between the core and external subdomains in the RBD is marked with a dashed line. The right panel (surface representation) was generated by rotating the structure in the left panel (cartoon representation) around a vertical axis by about $90^{\circ} . \mathbf{f}$, The real-time binding profile between our purified RBD protein and ACE2 characterized using surface plasmon resonance (Biacore).

all of the sites were found to be distant from the bound ACE2 (Fig. 1e), indicating that decorated glycans may not interfere with receptor recognition and/or binding.

The binding of our RBD protein with ACE2 was then confirmed using surface plasmon resonance (Biacore). In agreement with a previous study $^{2}$, potent interactions were observed. The binding affinity was calculated to be about $K_{\mathrm{d}}=1.54 \mathrm{nM}$ (dissociation constant), with an association rate constant $\left(k_{\mathrm{a}}\right)=1.33 \times 10^{7} \mathrm{M}^{-1} \mathrm{~s}^{-1}$ and a dissociation rate constant $\left(k_{\mathrm{d}}\right)$ of $2.05 \times 10^{-2} \mathrm{~s}^{-1}$ (Fig. 1f). This finding shows that our RBD binds to ACE2 with a high affinity, which is a good reflection of the native conformation of our RBD protein.

\section{Identification of antibodies against the RBD}

First, aluminium precipitation was selected to serve as a vaccine adjuvant on the basis of its effectiveness and safety ${ }^{11,12}$. In this study, all vaccine preparations were prepared by the addition of aluminium hydroxide gel to various proteins, which results in the aluminium precipitation of protein vaccine candidates.

Mice were immunized with a range of different doses $(0.1-20 \mu \mathrm{g})$ and regimens. For example, mice were immunized with a single injection on day 0 and sera were collected on day 7; with two vaccinations on days 0 and 7 , and sera were collected on day 21 , or with two doses on days 0 
and 14 and sera were collected on day 21 . In some experiments, a third dose was also given on day 21 . To assess the humoral immune responses induced by the recombinant RBD, we used an enzyme-linked immunosorbent assay (ELISA) for RBD-specific antibodies. Given the urgent need for an effective vaccine globally, special attention was provided to the early antibody response and its ability to neutralize SARS-CoV-2.

Sera obtained on day 7 after the first dose of the candidate vaccine already showed elevated IgG and IgM responses to the recombinant RBD (Fig. 2a). By contrast, the sera from pre-immunization and those from control mice treated with phosphate-buffered saline (PBS) or aluminium gel showed only background-level antibody responses. Furthermore, the antibody reaction was dose-dependent, and could be induced with a very low dose of the vaccinated protein $(0.1 \mu \mathrm{g}$ per mouse) 7 days after administration (Fig. 2b). The recombinant RBD protein alone was already effective in inducing the production of specific antibodies; however, addition of the aluminium adjuvant significantly enhanced the induction of antibodies with a higher level of specific antibodies by day 7 (Fig. 2a) and an even higher level of specific antibodies by day 21 (Fig. 2c). The sera obtained 9 days after the third vaccination (booster dose given on day 21) showed a very strong specific antibody response in the mice (Fig. $2 \mathrm{~d}$ ), with a positive reaction at a dilution of 1:204,800.

The antibody reaction was also dose-dependent (Fig. 2b). This dose-dependent response was also observed in rabbits, and a substantial level of specific antibody could be induced with a low dose of $1 \mu \mathrm{g}$ per rabbit per injection and with three doses of the candidate vaccines given ( $n=40$ rabbits) (Extended Data Fig. 1a). In addition, an elevated IgM antibody response could be observed as early as 7 days after the first vaccination (Extended Data Fig. 1c). Notably, we also tested the viral neutralization activity of the sera challenged in vitro with a pseudovirus and a high level of activity was observed (see 'Characterization of the sera from immunized animals') (Extended Data Fig. 1b)

We also tested our candidate vaccine in non-human primates. Ten monkeys were vaccinated on days 0 and 7 and immune sera were obtained at day 7 after each vaccination with our candidate vaccine; pre-immunization sera were used as a reference. Sera from days 7 and 14 after immunization showed a significantly elevated IgG response to the recombinant RBD and had an increased level of neutralizing antibodies against the pseudovirus (Extended Data Fig. 2). In addition, neutralizing antibodies against live SARS-CoV-2 were obtained after the vaccination of non-human primates and subsequent challenge with live SARS-CoV-2 (see 'Prevention of infection in non-human primates') (Fig. 3c).

The antigenicity of the recombinant RBD was also tested for seroreactivity using human sera that were obtained from healthy individuals and patients with COVID-19. Sera from 20 healthy donors and 16 patients with COVID-19 were tested for their IgG and IgM responses to the recombinant RBD. All 16 patients with COVID-19 showed significantly elevated levels of IgG and IgM against the recombinant RBD, compared with the background signal recorded using sera of the healthy donors (Fig. 2e).

\section{Characterization of the sera from immunized animals}

In the next sets of experiments, sera from immunized animals were tested for the blocking activity of the RBD binding to ACE2 receptor. RBD-ACE2 positivity was detected-in the absence of immune sera-in 90.2\% of Huh7 cells, which were used as positive control (Fig. 3a). With the immunized sera obtained from mice 7 days after a single dose $(5 \mu \mathrm{g})$ of the candidate vaccine, only $14.3 \%$ of Huh7 cells were RBD-ACE2 positive. Sera from mice treated with $P B S$ at the same dilution had almost no inhibitory activity, and $87.4 \%$ of cells were RBD-ACE2 positive (Fig. 3a). These findings indicate that the sera from early vaccination with a single dose $(5 \mu \mathrm{g})$ in mice could effectively block the binding of the RBD to the ACE2 receptor in human cells.

A neutralization assay using a pseudovirus is regarded to be a sensitive and quantitative method for assessing the neutralization of
SARS-CoV ${ }^{13}$. Immune sera from non-human primates, mice and rabbits were tested for neutralizing activity against a SARS-CoV-2 pseudovirus using HEK293T cells that expressed ACE2. Immune sera from the non-human primates 7 days after the first vaccination can almost completely block infection with the SARS-CoV-2 pseudovirus (Fig. 3b). Similarly, almost-complete neutralization of the SARS-CoV-2 pseudovirus was observed using the immune sera from mice and rabbits 14 days after the first vaccination (Extended Data Fig. 3a, b). In addition, the immune sera from both mice bearing a human ACE2 transgene and wild-type mice 14 days after the second vaccination showed a similar level of neutralizing antibodies with a calculated dilution of 1:32 or lower that can completely protect Vero E6 cells from live SARS-CoV-2 infection (Extended Data Fig. 4). When immune sera from rabbits immunized with 3 doses of the vaccine candidate were tested, we found that the viral neutralization activity with $50 \%$ neutralization $\left(\mathrm{EC}_{50}\right)$ was at a dilution of 1:2,826 in rabbits (Extended Data Fig. 1b).

\section{Prevention of infection in non-human primates}

In the subsequent experiments, we also tested whether the RBD vaccine could block the infection in non-human primates inoculated with live SARS-CoV-2. We immunized non-human primates with two intramuscular injections on days 0 and 7 of $20 \mu$ g or $40 \mu$ g per dose and then challenged the monkeys with live SARS-CoV-2 viruses 28 days after the first vaccination. The control groups included treatment with PBS or aluminium hydroxide gel alone. Neutralizing antibodies against live SARS-CoV-2 were detected in all vaccinated non-human primates, whereas no neutralizing antibodies were detected in the two control groups (Fig. 3c).

A quantitative real-time reverse-transcription PCR (RT-qPCR) assay was used to measure viral genomic RNA (gRNA) and viral subgenomic RNA (sgRNA). The expression of sgRNA is indicative of viral replication. Lung tissues of non-human primates were collected on day 7 after challenge to determine the viral replication status. Lung tissues from the control groups showed high levels of viral gRNA and sgRNA copies. By contrast, no detectable viral gRNA or sgRNA was present in the vaccinated groups that received $20 \mu \mathrm{g}$ and $40 \mu \mathrm{g}$ of the vaccine with adjuvant (Fig. 3d). In addition, peak loads of viral gRNA were observed in throat swabs from the control monkeys (Fig. 3e) 3 days after inoculation. The peak loads were reduced after the vaccination; only around 1.6 and $3.8 \mathrm{ppm}$ of the viral loads were found in the monkeys vaccinated with $20 \mu \mathrm{g}$ and $40 \mu \mathrm{g}$ of the vaccine, respectively, compared with the viral loads of the control group. Notably, no sgRNA was detected in the throat swabs of monkeys vaccinated with either the $20-\mu \mathrm{g}$ or $40-\mu \mathrm{g}$ dose after challenge with SARS-CoV-2 (Fig. 3e), whereas high levels of sgRNA were observed in the control monkeys. Peak levels of viral gRNA and sgRNA in anal swabs were observed in the control groups 5 and 6 days after inoculation, whereas only very low levels of gRNA were detected in the vaccinated monkeys and no sgRNA was detected in the anal swabs of non-human primates vaccinated with either the 20- $\mu$ g or 40- $\mu$ g dose (Fig. 3f). These data indicated that the low level of gRNA that was detected was due to the high-inoculation dose-which may have been neutralized already-and there is no evidence of viral replication, no sgRNA was detected.

The lung tissues of monkeys from the two control groups (PBS or aluminium hydroxide alone) showed typical histopathological changes of viral interstitial pneumonia, a key feature of COVID-19. As shown in Fig. 3g, the microscopy findings included apparent thickened alveolar walls and heavy interstitial infiltrates of mononuclear inflammatory cells. The alveolar space also showed heavy infiltration of mononuclear inflammatory cells and exudates with the disappearance of recognizable architecture. In addition, diffuse haemorrhage and type-II pneumocyte hyperplasia was also observed. By contrast, lung tissues from non-human primates vaccinated with the RBD vaccine $(20 \mu \mathrm{g}$ or $40 \mu \mathrm{g})$ showed no significant histopathological changes and appeared to be normal (Fig. 3g). 

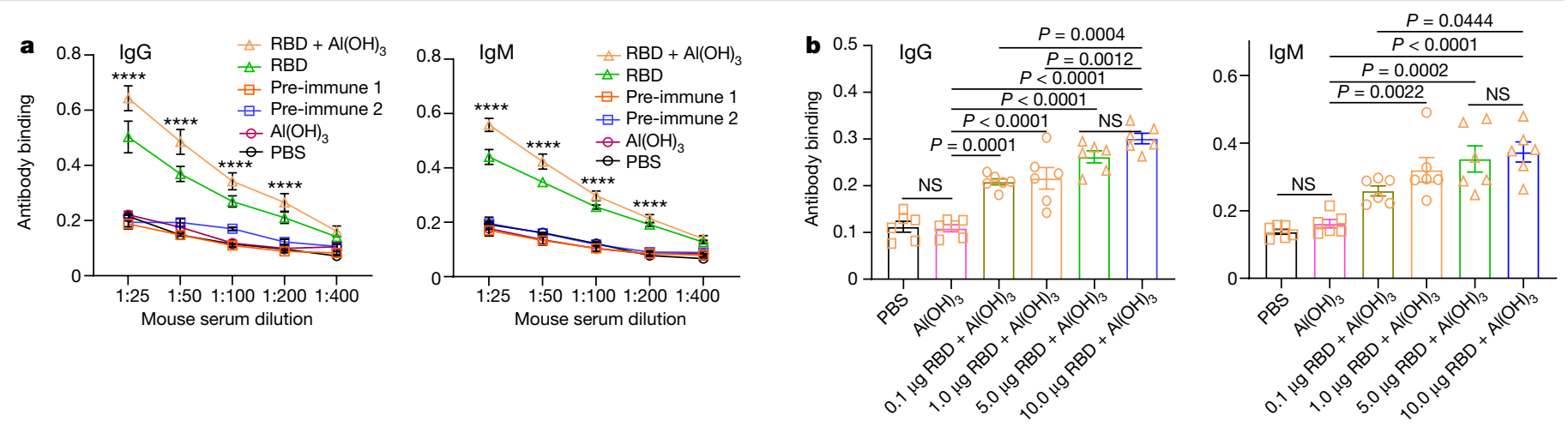

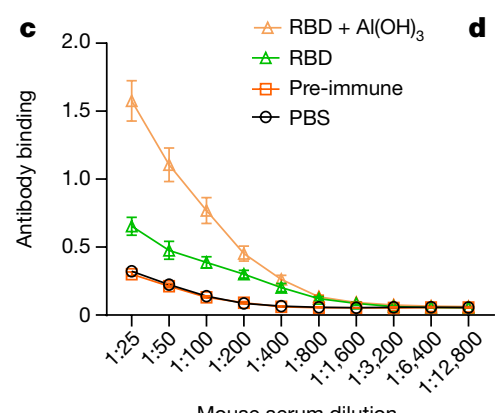

Mouse serum dilution

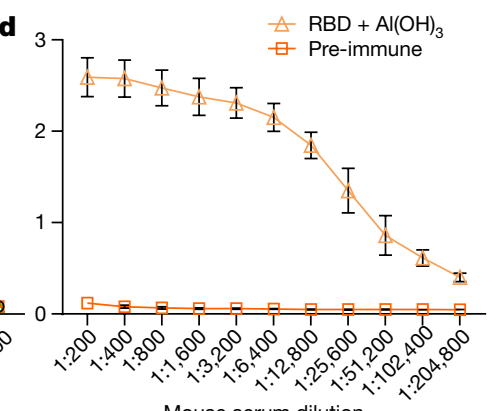

Mouse serum dilution

Fig. 2 Serum antibody response against the $R B D$ of the spike protein in patients and mice. a, The mice were immunized with $5 \mu \mathrm{g}$ recombinant RBD protein per mouse in $50 \mu \mathrm{l}$ in the presence of aluminium hydroxide $(\mathrm{RBD}+\mathrm{Al})$, compared with the control groups that received the recombinant RBD protein aluminium hydroxide $\left(\mathrm{Al}(\mathrm{OH})_{3}\right)$ or PBS alone or with the pre-immune groups. Sera were collected from the mice 7 days after the first dose of vaccine and the levels of IgG and IgM against the recombinant RBD protein were tested for different serum dilutions using ELISA (Methods). Serum antibody binding was measured as absorbance at $450 \mathrm{~nm}$. Data are mean \pm s.e.m. of five mouse sera per group. $P$ values were determined by two-way analysis of variance (ANOVA). $P$ values are shown for the $\mathrm{RBD}+\mathrm{Al}(\mathrm{OH})_{3}$ group versus the $\mathrm{Al}(\mathrm{OH})_{3}, \mathrm{PBS}$ or pre-immune groups. ${ }^{* * *} P<0.0001$. Comparison between $\mathrm{RBD}+\mathrm{Al}(\mathrm{OH})_{3}$ and the RBD alone for IgG, $P<0.0001$ and $P<0.002$ for the dilution of 1:25 and 1:50, respectively. Similar results were found in three independent experiments ( $n=5$ mice per group). b, The mice were immunized with 0.1-10 $\mu \mathrm{g}$ recombinant

\section{The immune pathways involved in RBD vaccination}

Next, the potential pathways through which our recombinant RBD protein vaccine acts to mount an immune humoral response were evaluated. As shown in Extended Data Fig. 5a, a reduction in the level of IgGs induced

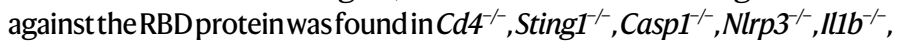
$\mathrm{Tlr}^{-/-}$and $\mathrm{Tlr}^{-/-}$mice compared with wild-type mice, whereas no effect on the level of IgG induction was found in other knockout mice $\left(\mathrm{Cd}^{8 \mathrm{a}^{-/}}\right)$.

Cellular immune responses could also be involved in the clearance of the SARS-CoV infection in which both CD4 and CD8 T cells are involved ${ }^{14-16}$. The lymphocytes isolated from vaccine-immunized mice induced increased levels of IFN $\gamma$ and IL-4 when stimulated with the recombinant RBD (Extended Data Fig.5b). Moreover, using flow cytometry, the number of the memory lymphocytes (including CD $4^{+} \mathrm{CD} 44^{\text {high }} \mathrm{IL}-4^{+}, \mathrm{CD} 4^{+} \mathrm{CD} 44^{\text {high }} \mathrm{IFN} \gamma^{+}$ and $\mathrm{CD} 8^{+} \mathrm{CD} 44^{\text {high }} \mathrm{IFN} \gamma^{+}$cells) was found to be increased in the mice treated with the candidate RBD vaccine (Extended Data Fig. $5 \mathrm{c}$ ).

To investigate whether the immune sera or the splenic T cells triggered by our vaccine had a role in the protection against live SARS-CoV-2, we performedadoptive therapy of immune sera or splenic T cells from vaccinated mice. Adoptive therapy of splenic T cells $\left(\mathrm{CD} 4^{+}\right.$and $\mathrm{CD} 8^{+}$cells) did not provide protectionagainst infection with SARS-CoV-2. By contrast, adoptive therapy of $0.1 \mathrm{ml}$ of the pooled sera fromimmunizedmiceshowed nodetectableviral replication (Fig. 4a), no significant histopathological changes (Extended Data Fig. 6a) and no weight loss (Extended Data Fig. 6b) in recipient mice,
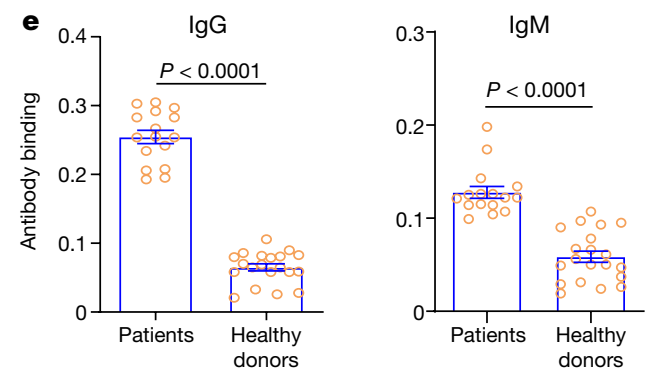

RBD protein per mouse in $50 \mu$ in the presence of $\mathrm{Al}(\mathrm{OH})_{3}$, or with $\mathrm{Al}(\mathrm{OH})_{3}$ or PBS alone. Sera were collected from the mice 7 days after the first dose of the vaccine and were tested at a 1:50 dilution for the levels of IgG and IgM against the RBD of the spike protein using ELISA. Data are mean \pm s.e.m. of six mouse serum samples per group. $P$ values were determined by one-way ANOVA. , Sera were collected 14 days after two vaccinations on days 0 and 7 with $5 \mu \mathrm{g}$ recombinantRBD protein per mouse in $50 \mu$ in the presence of aluminium hydroxide or with recombinant RBD protein alone, and the IgG level in sera was tested at the indicated dilutions using ELISA. Similar results were obtained in three independent experiments.d, Sera were collected 9 days after the three vaccinations on days 0,14 and 21 using the same doses as c.e, Serum samples were collected from 16 patients who were infected with SARS-CoV-2 and had recovered, and from 20 healthy donors. Antibody binding to the RBD was detected using 1:5 diluted sera by ELISA (Methods). Data are mean \pm s.e.m. $P$ values were determined by one-way ANOVA.

compared with mice treated with PBS as a control. Even the sera from mice 7 days aftera single dose of the vaccine could completely protectmiceagainst infection with live SARS-CoV-2 (Extended Data Fig. 6c-e). In addition, no evidence of antibody-dependentenhancement or acceleration of pneumonia was observed as no mice that received the RBD-vaccinate immune sera developed any evidence of pneumonia. Considering the important role of the antibody for the protective immunity shown in the present study, we also tested the other forms of the spike protein for the optimal induction of neutralizing antibodies, and found that the recombinantRBDvaccinehada much higher viral neutralization activity compared with vaccinescomprising theextracellulardomain orS1subunit(Fig.4b). Noviral neutralizationactivity was found using a vaccine comprising the 22 subunit (Fig. 4b).

Although increased levels of IFN $\gamma$ and IL-4 produced by lymphocytes stimulated with the RBD in vitro were observed, there were no significant increase in these two and other inflammatory cytokines in the plasma of the mice immunized with the RBD vaccine (Extended Data Fig. $5 \mathrm{~d}$ ), indicating that the RBD did not induce a systemic inflammatory reaction.

To further address the safety of the candidate vaccine, the potential toxicity of the vaccine was evaluated in the non-human primates $(n=50)$ in compliance with good laboratory practices. No adverse events were observed in parameters such as body weight, ruffling of fur, behavioural changes or appetite. No pathological changes were observed in the liver, lung, kidney, spleen, brain, heart or other tissues of the primates when investigated using microscopy. No changes 

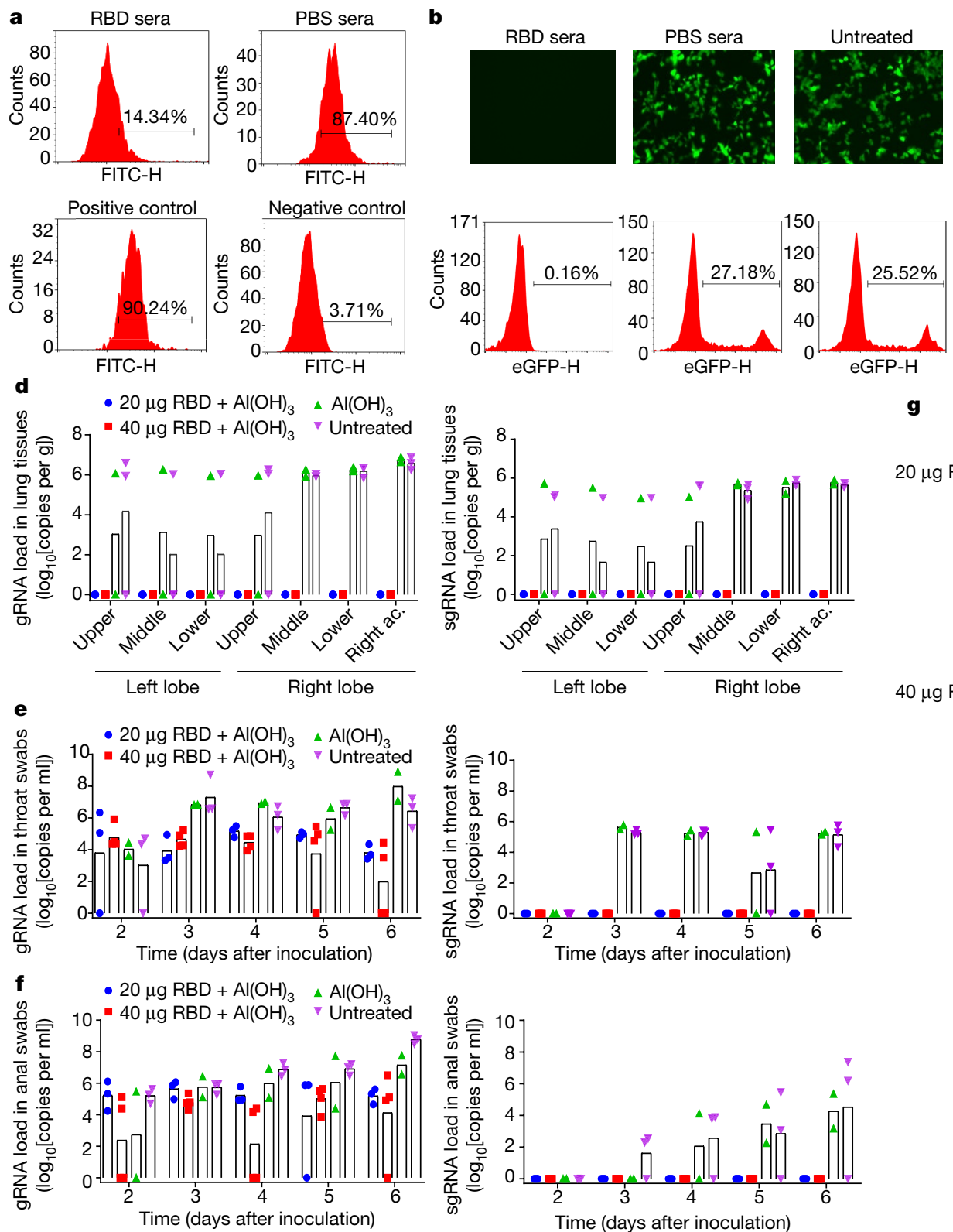

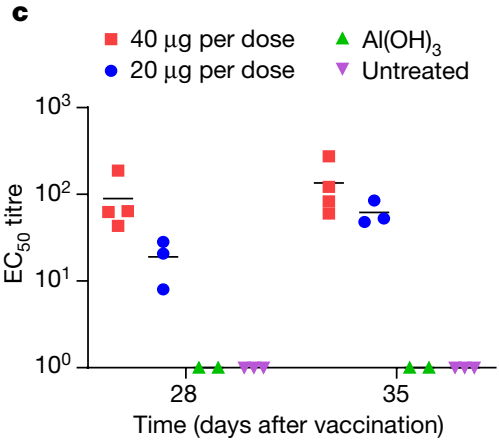

\section{$\mathbf{g}$}
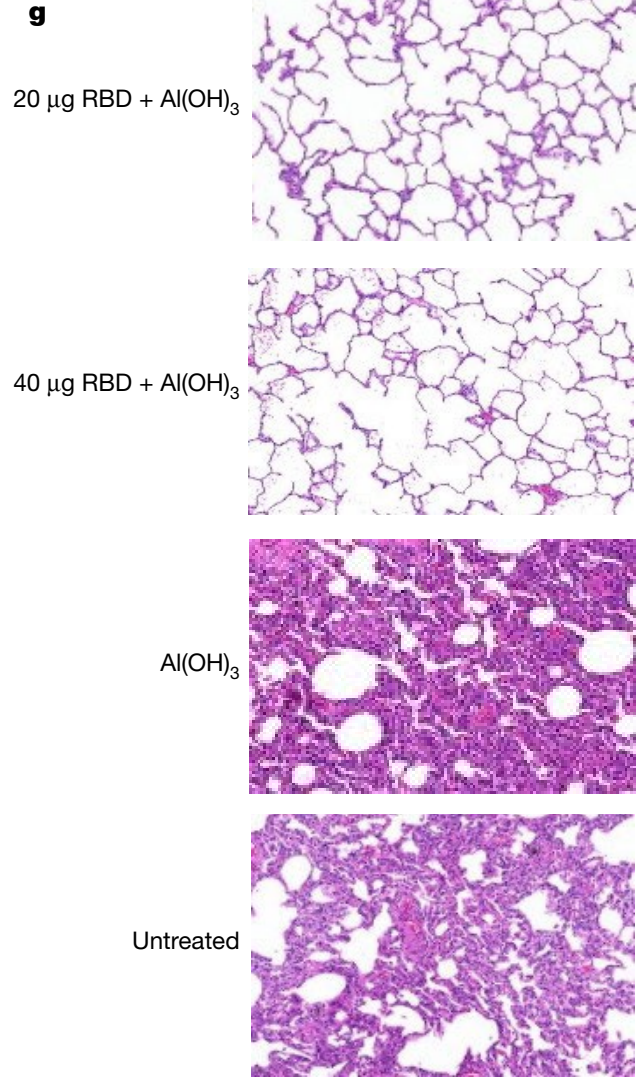

Fig. 3 Functional characterization of the sera from the immunized animals and the protection of the non-human primates from SARS-CoV-2 infection. a, Inhibition of the binding of the RBD to ACE2. A recombinant SARS-CoV-2 RBD-Fc fusion protein was added to $\mathrm{ACE}^{+} \mathrm{Huh} 7$ cells in the presence or absence of sera at a dilution of 1:5, followed by incubation with an anti-human IgG-FITC conjugated antibody. The following treatments were included: RBD sera (sera from mice immunized with the RBD vaccine on day 7 after the first vaccination); PBS sera (sera from mice treated with PBS as a control); positive control (without sera); and negative control (cells stained with anti-human IgG-FITC conjugated antibody alone). b, Neutralization of infection with the SARS-CoV-2 pseudovirus by sera from non-human primates (magnification, 200×). The following treatments were included: RBD sera (sera from non-human primates immunized with RBD); PBS sera (sera from non-human primates treated with
PBS as a control); untreated (infection with SARS-CoV-2 pseudovirus without sera).c-g, Non-human primates were immunized on days 0 and 7 with two intramuscular injections of $20 \mu \mathrm{g}$ or $40 \mu \mathrm{g}$ per dose and then challenged with SARS-CoV-2 intranasally $\left(0.5 \mathrm{ml}, 10^{6}\right.$ plaque-forming units (PFU) $\left.\mathrm{ml}^{-1}\right)$ on day 28 after the first vaccination (Methods). c, Neutralization of live SARS-CoV-2 infection by the immune sera from non-human primates was measured (Methods). d-f, RT-qPCR was used to measure gRNA and sgRNA in lung tissues (d), throat swabs (e) and the anal swabs (f). Data are individual values and geometric means. ac. indicates accessory lobes. The following treatment groups were included: $40 \mu \mathrm{g} \mathrm{RBD}+\mathrm{Al}(\mathrm{OH})_{3}, n=4 ; 20 \mu \mathrm{g} \mathrm{RBD}+\mathrm{Al}(\mathrm{OH})_{3}, n=3$; untreated, $n=3 ; \mathrm{Al}(\mathrm{OH})_{3}, n=2$. g, Histopathological changes in lung tissues observed by light microscopy using haematoxylin and eosin staining (magnification, 200×). in peripheral blood counts and differentials were found (data not shown).

\section{Discussion}

SARS-CoV-2 is the cause of a major pandemic and an effective vaccine is urgently needed. It has been reported that several vaccines including inactivated viruses, adenoviral vectors that contained the spike protein or RNA vaccines have been tested in monkeys or in clinical trials ${ }^{17-20}$. The fact that our candidate RBD vaccine can induce effective virus-neutralizing activity in three animal species is very encouraging. It is also encouraging to observe (1) that the antibodies shared common binding epitopes with patients with COVID-19; (2) that there was strong virus-neutralizing activity associated with a good humoral response; (3) that a simple vaccine adjuvant such as aluminium could further enhance the immune response; (4) that even a single dose of the vaccine could already generate a high 


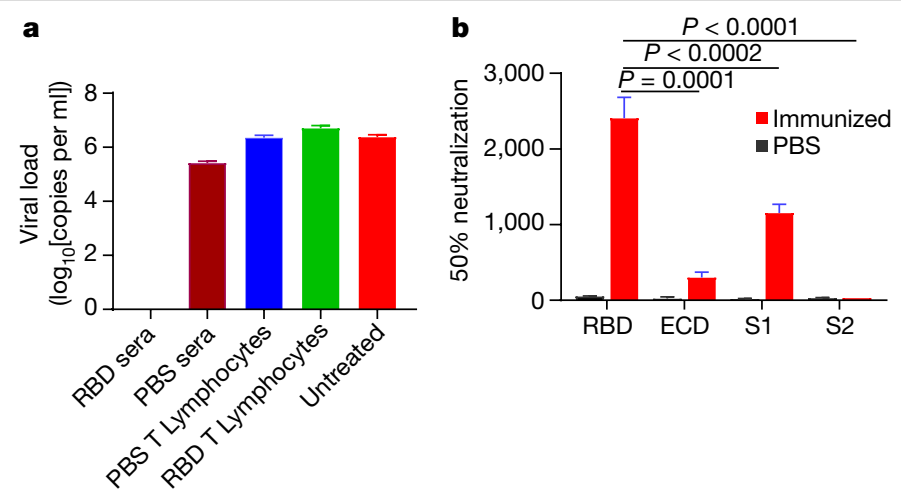

Fig. 4 | The role of immune sera and splenic $T$ cells in the protective immunity in vaccinated mice. a, Human $A C E 2$ (hACE2) transgene-bearing mice with a $\mathrm{C} 57 \mathrm{BL} / 6$ background received $0.1 \mathrm{ml}$ of pooled sera or $5 \times 10^{7}$ splenic T cells isolated from wild-type mice of the same $\mathrm{C} 57 \mathrm{BL} / 6$ background 9 days after the third dose of the candidate vaccine or from mice treated with PBS as a control. The mice were euthanized 5 days after the challenge with live virus, and viral replication in lung tissues was evaluated. b, Infection of HEK293 cells that express human ACE2 by SARS-CoV-2 pseudovirus was determined in the presence of mouse sera at a series of threefold dilutions (Methods). The mice were immunized with $5 \mu \mathrm{g}$ recombinant $\mathrm{RBD}$ (amino acids 319-545), the extracellular domain (ECD, amino acids $16-1213$ ), the $S 1$ subunit ( $\mathrm{S} 1$, amino acids 16-685) or S2 subunit (S2, amino acids 686-1213) in the presence of aluminium hydroxide gel. The $50 \%$ neutralization data are mean \pm s.e.m.

level of virus-neutralizing activity; (5) that the vaccine could protect non-human primates against a challenge with live SARS-CoV-2; and (6) that the toxicology studies in the non-human primates showed that this vaccine candidate is safe to use. Furthermore, our vaccine strategy based on the RBD has been supported by recent findings that most of the neutralizing activity is directed against the RBD in the sera of patients with COVID-19 and by evidence from human monoclonal antibodies ${ }^{20-22}$. In addition, this recombinant RBD protein may be technically and commercially feasible to manufacture at a global supply scale. All of these features are excellent prospects that support the further development of this vaccine candidate.

The early protective virus-neutralizing activity that was observed is also notable. Given the current pandemic, developing some type of virus-neutralizing activity around 7 days after the first dose would be very helpful (while still following the final vaccination regimen as defined clinically); this effect may have a strong effect on the treatment of individuals with COVID-19 and the prevention of the spread of SARS-COV-2 in the population. Although we found no evidence of enhancement in the passive transfer model in mice or after viral challenge in the immunized non-human primates, this does not rule out the possibility of the enhancement by antibodies or cellular immunity after vaccination in other species, including humans.

In summary, our findings highlight the importance of the RBD domain of the spike protein in SARS-CoV-2 vaccine design and provide a rationale for the development of a protective vaccine through the induction of antibodies against the RBD domain.

\section{Online content}

Any methods, additional references, Nature Research reporting summaries, source data, extended data, supplementary information, acknowledgements, peer review information; details of author contributions and competing interests; and statements of data and code availability are available at https://doi.org/10.1038/s41586-020-2599-8.

1. Zhou, P. et al. A pneumonia outbreak associated with a new coronavirus of probable bat origin. Nature 579, 270-273 (2020).

2. Wrapp, D. et al. Cryo-EM structure of the 2019-nCoV spike in the prefusion conformation. Science 367, 1260-1263 (2020).
3. Li, Q. et al. Early transmission dynamics in Wuhan, China, of novel coronavirus-infected pneumonia. N. Engl. J. Med. 382, 1199-1207 (2020).

4. Huang, C. et al. Clinical features of patients infected with 2019 novel coronavirus in Wuhan, China. Lancet 395, 497-506 (2020).

5. Contreras-Gómez, A., Sánchez-Mirón, A., García-Camacho, F., Molina-Grima, E. \& Chisti, Y. Protein production using the baculovirus-insect cell expression system. Biotechnol. Prog. 30, 1-18 (2014).

6. Cox, M. M. \& Hashimoto, Y. A fast track influenza virus vaccine produced in insect cells. J. Invertebr. Pathol. 107, S31-S41 (2011).

7. Lu, G. et al. Molecular basis of binding between novel human coronavirus MERS-CoV and its receptor CD26. Nature 500, 227-231 (2013).

8. Wang, Q. et al. Bat origins of MERS-CoV supported by bat coronavirus HKU4 usage of human receptor CD26. Cell Host Microbe 16, 328-337 (2014).

9. Xiao, K. \& Tian, Z. GPSeeker enables quantitative structural N-glycoproteomics for siteand structure-specific characterization of differentially expressed $\mathrm{N}$-glycosylation in hepatocellular carcinoma. J. Proteome Res. 18, 2885-2895 (2019).

10. Wang, Y., Xu, F., Xiao, K., Chen, Y. \& Tian, Z. Site- and structure-specific characterization of $\mathrm{N}$-glycoprotein markers of MCF-7 cancer stem cells using isotopic-labelling quantitative N-glycoproteomics. Chem. Commun. 55, 7934-7937 (2019).

11. MacLeod, M. K. et al. Vaccine adjuvants aluminum and monophosphoryl lipid A provide distinct signals to generate protective cytotoxic memory CD8 T cells. Proc. Natl Acad. Sci. USA 108, 7914-7919 (2011).

12. Luo, M. et al. Simultaneous enhancement of cellular and humoral immunity by the high salt formulation of $\mathrm{Al}(\mathrm{OH})_{3}$ adjuvant. Cell Res. 27, 586-589 (2017).

13. Wong, S. K., Li, W., Moore, M. J., Choe, H. \& Farzan, M. A 193-amino acid fragment of the SARS coronavirus $S$ protein efficiently binds angiotensin-converting enzyme 2. J. Biol. Chem. 279, 3197-3201 (2004).

14. Yang, Z. Y. et al. A DNA vaccine induces SARS coronavirus neutralization and protective immunity in mice. Nature 428, 561-564 (2004).

15. Guo, Y. et al. Elicitation of immunity in mice after immunization with the $\mathrm{S} 2$ subunit of the severe acute respiratory syndrome coronavirus. DNA Cell Biol. 24, 510-515 (2005).

16. He, Y. et al. Cross-neutralization of human and palm civet severe acute respiratory syndrome coronaviruses by antibodies targeting the receptor-binding domain of spike protein. J. Immunol. 176, 6085-6092 (2006).

17. van Doremalen, N. et al. ChAdOx1 nCoV-19 vaccine prevents SARS-CoV-2 pneumonia in rhesus macaques. Nature https://doi.org/10.1038/s41586-020-2608-y (2020).

18. Zhu, F. C. et al. Safety, tolerability, and immunogenicity of a recombinant adenovirus type- 5 vectored COVID-19 vaccine: a dose-escalation, open-label, non-randomised, first-in-human trial. Lancet 395, 1845-1854 (2020).

19. Gao, Q. et al. Development of an inactivated vaccine candidate for SARS-CoV-2. Science 369, 77-81 (2020).

20. Dai, L. et al. A universal design of betacoronavirus vaccines against COVID-19, MERS, and SARS. Cell 182, 722-733 (2020).

21. Robbiani, D. F. et al. Convergent antibody responses to SARS-CoV-2 in convalescent individuals. Nature 584, 437-442 (2020).

22. Wec, A. Z. et al. Broad neutralization of SARS-related viruses by human monoclonal antibodies. Science 369, 731-736 (2020)

Publisher's note Springer Nature remains neutral with regard to jurisdictional claims in published maps and institutional affiliations.

(c) The Author(s), under exclusive licence to Springer Nature Limited 2020

'Laboratory of Aging Research and Cancer Drug Target, State Key Laboratory of Biotherapy and Cancer Center, National Clinical Research Center for Geriatrics, West China Hospital, Sichuan University, Chengdu, China. ${ }^{2}$ National Kunming High-level Biosafety Primate Research Center, Institute of Medical Biology, Chinese Academy of Medical Sciences and Peking Union Medical College, Yunnan, China. ${ }^{3}$ Key Laboratory of Human Disease Comparative Medicine, Chinese Ministry of Health, Beijing Key Laboratory for Animal Models of Emerging and Remerging Infectious Diseases, Institute of Laboratory Animal Science, Chinese Academy of Medical Sciences and Comparative Medicine Center, Peking Union Medical College, Beijing, China. ${ }^{4}$ National Institutes for Food and Drug Control (NIFDC), Beijing, China. ${ }^{5}$ National Chengdu Center for Safety Evaluation of Drugs, State Key Laboratory of Biotherapy and Cancer Center, West China Hospital, Sichuan University, Collaborative Innovation Center for Biotherapy, Chengdu, China. ${ }^{6}$ Shanghai Key Laboratory of Chemical Assessment and Sustainability, School of Chemical Science \& Engineering, Tongji University, Shanghai, China. ${ }^{7}$ Treatment and Research Center for Infectious Diseases, The Fifth Medical Center of PLA General Hospital, National Clinical Research Center for Infectious Diseases, Beijing, China. ${ }^{8}$ Center for Biomedicine and Innovations, Faculty of Medicine, Macau University of Science and Technology, Macau, China. ${ }^{9}$ Department of Computer Science and Technology, Tsinghua University, Beijing, China. ${ }^{10}$ Department of Applied Biology and Chemical Technology, Hong Kong Polytechnic University, Hong Kong, China. "Sichuan Provincial Key Laboratory for Human Disease Gene Study and Institute of Laboratory Medicine, Sichuan Provincial People's Hospital, University of Electronic Science and Technology of China, Chengdu, China. ${ }^{12}$ State Key Laboratory of Medical Molecular Biology, Chinese Academy of Medical Sciences, School of Basic Medicine, Peking Union Medical College, Beijing, China. ${ }^{13}$ Emergency Department, State Key Laboratory of Biotherapy, West China Hospital, Sichuan University, Chengdu, China. ${ }^{14}$ These authors contributed equally: Jingyun Yang, Wei Wang, Zimin Chen, Shuaiyao Lu, Fanli Yang, Zhenfei Bi, Linlin Bao.

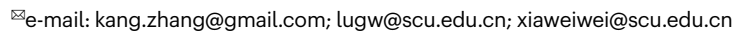




\section{Methods}

\section{Data reporting}

No statistical methods were used to predetermine sample size. The experiments were not randomized and the investigators were not blinded to allocation during experiments and outcome assessment.

\section{Bacterial strains and cell lines}

All Escherichia coli strains were cultured in lysogeny broth medium $(1 \% \mathrm{w} / \mathrm{v}$ tryptone, $0.5 \% \mathrm{w} / \mathrm{v}$ yeast extract and $1 \% \mathrm{w} / \mathrm{v} \mathrm{NaCl})$ using a non-humidified shaker at $37^{\circ} \mathrm{C}$. Spodoptera frugiperda (Sf9) cells and Trichoplusia ni $(\mathrm{Hi}$ ) cells were maintained in SIM SF medium and the SIM HF medium (Sino Biological), respectively, using a non-humidified shaker at $27^{\circ} \mathrm{C}$. HEK293T and Huh-7 cells were maintained in Dulbecco's modified Eagle's medium (DMEM) supplemented with $10 \%$ fetal bovine serum (FBS), 100 units of penicillin and $0.1 \mathrm{mg} / \mathrm{ml}$ of streptomycin with $5 \% \mathrm{CO}_{2}$ at $37^{\circ} \mathrm{C}$.

\section{Gene cloning, protein expression and purification}

For antigenicity evaluation, the RBD of the spike protein of SARS-CoV-2 was expressed using the Bac-to-Bac baculovirus expression system (Invitrogen). The coding sequence (codon optimized for insect cells) for the RBD region, which spans residues 319-545 of the spike of the SARS-CoV-2 Wuhan-Hu-1 isolate (GenBank accession number MN908947), was synthesized by Convenience Biology. For gene cloning, a previously described gp67 signal peptide sequence ${ }^{7}$ was first incorporated into the $\mathrm{pFastBac1} \mathrm{vector} \mathrm{via} \mathrm{the} \mathrm{BamHI} \mathrm{and} \mathrm{EcoRI} \mathrm{restric-}$ tion sites. The RBD gene was then subcloned into the modified vector via the EcoRI and HindIII sites. In addition, an $8 \times \mathrm{His}$ tag was further added to the protein $\mathrm{C}$ terminus to facilitate protein purification. The sequencing-verified plasmid was subsequently transformed into E. coli $\mathrm{DH} 10$ b cells to generate the recombinant bacmids.

For protein expression, the bacmid was first transfected into Sf9 insect cells using Lipolnsect Transfection Regent (Beyotime Biotechnology) according to the manufacturer's instructions. The cell culture supernatants, which contain the packaged recombinant baculoviruses, were collected about $72 \mathrm{~h}$ after transfection. The baculovirus was then passaged in Sf9 cells two to three times before being used for protein production in Sf9 cells.

For protein purification, the culture supernatants from the insect cells were collected about $72 \mathrm{~h}$ after infection and passed through a 5-ml HisTrap excel column (GE Healthcare) for primary purification. The recovered proteins were further purified on a Superdex 200 Increase 10/300 GL column (GE Healthcare). Finally, the proteins were exchanged into a buffer consisting of $20 \mathrm{mM}$ Tris- $\mathrm{HCl}(\mathrm{pH} 8.0)$ and $150 \mathrm{mM} \mathrm{NaCl}$ for further use. Tag-free RBD protein (amino acids 320-545) was also prepared. The purity of the protein was determined by SDS-PAGE and visualized by staining with Coomassie blue and by western blotting using the rabbit anti-RBD antibody (Sino Biological).

Following the protocol described above, the ECD (extracellular domain, amino acids 16-1213), S1 (amino acids 16-685) and S2 (amino acids 686-1213) domain proteins of SARS-CoV-2 were prepared using the Bac-to-Bac baculovirus expression system. In brief, the individual

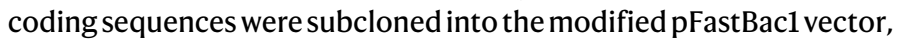
transposed into the bacmid in $E$. coli DH1Ob and transfected into insect cells for recombinant baculovirus package and protein expression. The target proteins were then purified via affinity chromatography to a purity, as determined by SDS-PAGE and Coomassie blue staining, similar to that of the RBD protein.

\section{Liquid chromatography-MS/MS analysis to identify glycosylation sites}

For MS analyses, the protein was first trypsin-digested as previously described $^{23,24}$. In brief, the purified RBD protein was precipitated with four volumes of precooled acetone in $-20^{\circ} \mathrm{C}$ overnight. The protein pellets were collected with centrifugation at 20,000 $g$ for $10 \mathrm{~min}$. After drying on ice, the protein pellets were dissolved in a denaturing buffer ( $5 \% 8 \mathrm{M}$ urea in $50 \mathrm{mM} \mathrm{v} / \mathrm{v} \mathrm{NH}_{4} \mathrm{HCO}_{3}$ ). The proteins were reduced with $20 \mathrm{mM} \mathrm{DTT}$ at $55^{\circ} \mathrm{C}$ for $60 \mathrm{~min}$, and alkylated with $55 \mathrm{mM}$ iodoacetamide in the dark at room temperature for an additional $30 \mathrm{~min}$. After carbamidomethylation, the proteins were digested with trypsin $(1: 50 \mathrm{w} / \mathrm{w})$ at $37^{\circ} \mathrm{C}$ overnight.

After being desalted with C18 ZipTip (Millipore) according to the manufacturer's instructions, the digested peptides were analysed by liquid chromatography (LC)-MS/MS using an EASY-nano-LC 1200 coupled to a Q-Exactive HF-X (Thermo Scientific) without the trap column. The peptide samples were loaded onto an in-house-packed reversed-phase C18 analytical column $(30 \mathrm{~cm}$ length $\times 360 \mu \mathrm{m}$ OD $\times 75 \mu \mathrm{m} \mathrm{ID}, 3 \mu \mathrm{m}$ particle, DIKMA) and were separated at a flow rate of $330 \mathrm{nl} / \mathrm{min}$. The column oven temperature was $60^{\circ} \mathrm{C}$. Buffer A was $0.2 \%$ formic acid in water, and buffer $\mathrm{B}$ was $80 \% \mathrm{ACN}$ with $0.2 \%$ formic acid. A 2-h gradient was applied with 3\% buffer B for 6 min, 3-48\% buffer B for 100 min, $48-100 \%$ buffer B for $6 \mathrm{~min}, 100 \%$ buffer B for $6 \mathrm{~min}$ and $2 \%$ buffer $B$ for the last $2 \mathrm{~min}$, as previously described ${ }^{25}$. MS spectra were acquired with $\mathrm{m} / z$ $700-2,000$ and a resolution of 60,000 at $m / z 200$. The automatic gain control was set at $2 \times 10^{5}$ with maximum fill time of $50 \mathrm{~ms}$. For MS/MS scans, the top 20 most-intense parent ions were selected with a $2.0 \mathrm{~m} / z$ isolation window and fragmented using high-energy collision dissociation with stepped normalized collision energies ${ }^{26}$ of $20 \%, 30 \%$ and $30 \%$. The automatic gain control value for MS/MS was set to a target value of $5 \times 10^{5}$, with a resolution of 3,000 and a maximum fill time of $250 \mathrm{~ms}$. Parent ions with a charge state of $z=1,8$ or unassigned were excluded and the intensity threshold was $3.3 \times 10^{4}$. The dynamic exclusion period was $20 \mathrm{~s}$. The temperature of the ion transfer capillary was set to $280^{\circ} \mathrm{C}$. The spray voltage was set to $2.8 \mathrm{kV}$.

For the identification of intact $\mathrm{N}$-glycopeptides, all the raw files were searched with GPSeeker 9 . To search matching precursor and fragment ions, the isotope peak abundance cut-off, isotope peak mass-to-charge ratio $(\mathrm{m} / \mathrm{z})$ deviation and isotope peak abundance deviation were, respectively, set to $40 \%, 20 \mathrm{ppm}$ and $50 \%$. The search of intact $\mathrm{N}$-glycopeptide spectrum matches included the following parameters: $\mathrm{Y} 1$ ion, Top4; the minimum percentage of matched fragment ions of the peptide backbone, $\geq 10 \%$; the minimum matched fragment ion of the $\mathrm{N}$-glycan moiety, $\geq 1$; TopN hits, $n=2$ with Top1 hit(s) having the lowest $P$ score; $G$-bracket, $\geq 1$; and GF score, $\geq 1$. The G-bracket for a given $\mathrm{N}$-glycosite is defined as the number of peptide backbone $\mathrm{b} / \mathrm{y}$ fragment ion pairs, each of which can independently localize to the $\mathrm{N}$-glycosite. The GF score for a given $\mathrm{N}$-glycan sequence structure is defined as the number of structure-diagnostic fragment ions, each of which can independently distinguish the structure from all the other putative structures with the same monosaccharide composition. After the database search of all the raw datasets, $\mathrm{N}$-glycopeptide spectrum matches were combined and intact $\mathrm{N}$-glycopeptides with the lowest $P$ score were chosen as the final IDs.

The raw MS files were further searched against the RBD sequence with SEQUEST in Proteome Discoverer (version 2.3; Thermo Fisher Scientific). The precursor peptide mass tolerance was $10 \mathrm{ppm}$ and the fragment ion mass tolerance was $0.02 \mathrm{Da}$. Two missing cleavages were allowed. Cysteine carbamidomethylation was set as a fixed modification. HexNAc (S/T), Hex(1)HexNAc(1) (S/T) and other potential O-glycosylation modifications with conventional oxidation of methionine and protein $\mathrm{N}$-terminal acetylation were set as variable modifications ${ }^{9,23}$. Percolator was performed with a false-discovery rate (FDR) of $1 \%$. All potential O-glycosylation sites were further manually confirmed by the $b$ ions and $y$ ions.

To determine the abundance of glycosylation, the number of MS/MS spectra of glycosylated peptides and their corresponding unmodified peptides were both counted. Glycosylation at different positions and glycosylation with different glycan isoforms on a peptide with identical sequence were combined. 


\section{Surface plasmon resonance analysis}

Surface plasmon resonance (SPR)-based measurements were performed by Biacore $8 \mathrm{~K}$ (GE Healthcare), as previously described ${ }^{2}$. Human ACE2-Fc was captured to around 100 response units (RUs) on Sensor Chip Protein A. For kinetic analysis, RBD protein was run across the chip in a twofold dilution series $(1,2,4,8,16,32 \mathrm{nM})$, with another channel set as control. Each sample that was bound to the antigen surface was dissociated by HBS-EP+ running buffer for $300 \mathrm{~s}$ at a flow rate of $30 \mu \mathrm{l} /$ min. Regeneration of the sensor chips was performed for $60 \mathrm{~s}$ using regeneration buffer (glycine $\mathrm{pH} 1.5)$. The association $\left(k_{\mathrm{a}}\right)$ and dissociation $\left(k_{\mathrm{d}}\right)$ rate constants, and the affinity value $\left(K_{\mathrm{d}}\right)$ were determined.

\section{Vaccine formulation and vaccinations of mice}

Aluminium-precipitated protein vaccines were prepared as previously described $^{27}$. In brief, the purified recombinant RBD protein was added at the different concentrations, mixed with aluminium hydroxide gel and incubated for $1 \mathrm{~h}$ at $5^{\circ} \mathrm{C}$. The different formulations were prepared with concentrations of $1-100 \mu \mathrm{g} / \mathrm{ml}$ for protein and $1.21 \mathrm{mg} / \mathrm{ml}$ for the aluminium hydroxide gel.

$\mathrm{BALB} / \mathrm{C}$ and $\mathrm{C} 57 \mathrm{BL} / 6$ mice (6-8 weeks of age) were injected intramuscularly with different doses $(0.1-20 \mu \mathrm{g}$ per mouse) of recombinant RBD and for different intervals. For example, mice were immunized with a single injection on day 0 and sera were collected on day 7 , with two vaccinations on days 0 and 7 and sera were collected on day 21, compared with two doses on days 0 and 14 and sera collected on day 21 . We also investigated the third vaccine on day 21 or longer. Additional control mice were injected with aluminium hydroxide adjuvant, recombinant RBD or PBS alone. Pre-immunization sera were collected before starting the immunization and sera were collected 7 days after each boost. We also immunized transgenic hACE2 mice with the RBD vaccine and found that the mice had similar level of the antibodies against RBD protein compared with wild-type mice (Extended Data Fig. 4).

Sera were kept at $4{ }^{\circ} \mathrm{C}$ before use. In an attempt to find the pathways that are activated by the recombinant RBD, the recombinant RBD

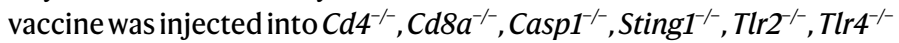
(all from Jackson Laboratory), $\mathrm{Nlrp3}^{-/-}$(from Genentech) and $/ 11 \mathrm{~b}^{-/-}$ (Tokyo University of Science) mice. All studies involving mice were approved by the Animal Care and Use Committee of Sichuan University.

\section{Vaccinations of rabbits}

For rabbit immunization, a total of 40 rabbits (4-6 months of age) were injected intramuscularly with different doses (1-40 $\mu \mathrm{g}$ per rabbit, $n=6$ rabbits per group) of recombinant RBD vaccine or with $20 \mu \mathrm{g}$ recombinant RBD protein alone $(n=6)$ on days 0,14 and 21 . We also immunized rabbits with aluminium hydroxide alone $(n=4)$ as a control group. The sera were collected 7 days after each vaccination. All studies involving rabbits were approved by the Animal Care and Use Committee of National Chengdu Center for Safety Evaluation of Drugs.

\section{Identification of serum antibodies against the RBD in patients and mice using an ELISA assay}

Blood samples were collected from the retro-orbital plexus of mice after each antigen boost. After coagulation at room temperature for 1-2 $\mathrm{h}$, blood samples were centrifuged at $3,000 \mathrm{rpm}$ for $10 \mathrm{~min}$ at $4{ }^{\circ} \mathrm{C}$. The upper serum layer was collected and stored at $-20^{\circ} \mathrm{C}$. Recombinant RBD (or S2 protein as a control) was used to coat flat-bottom 96-well plates (NUNC-MaxiSorp, Thermo Fisher Scientific) at a final concentration of $1 \mu \mathrm{g} / \mathrm{ml}$ in $50 \mathrm{mM}$ carbonate coating buffer ( $\mathrm{pH} 9.6$ ) at $4{ }^{\circ} \mathrm{C}$ overnight. The next day, plates were washed three times with PBS containing $0.1 \%$ Tween-20 (PBST), and blocking solution containing 1\% BSA in PBST was added, followed by $1 \mathrm{~h}$ incubation at room temperature. Serially diluted mouse sera were added and incubated at $37^{\circ} \mathrm{C}$ for $1 \mathrm{~h}$, and then the plates were washed three times with PBST. Antibodies, including goat anti-mouse IgG horseradish peroxidase (HRP)-conjugated antibody and anti-mouse IgG1/IgM HRP-conjugated antibody, were diluted 1:5,000 in blocking solution and added to the wells $(100 \mu \mathrm{l} /$ well $)$. After incubation for $1 \mathrm{~h}$ at room temperature, the plates were washed five times with PBST and developed with 3,3',5,5'-tetramethylbiphenyldiamine (TMB) for $10 \mathrm{~min}$. The reactions were stopped with $50 \mu \mathrm{l} /$ well of $1.0 \mathrm{M} \mathrm{H}_{2} \mathrm{SO}_{4}$ stop solution. The absorbance was measured on a microplate reader at $450 \mathrm{~nm}$. To measure the titre of RBD-specific antibodies induced by recombinant proteins, serum samples were serially diluted and measured by titration.

These studies were approved by the institutional ethics board of Sichuan Provincial People's Hospital. Data collection and analysis of patients were also required by the National Health Commission of the People's Republic of China to be part of a continuing public health outbreak investigation. To investigate the potential immunogenicity of the RBD of the S protein as a vaccine in humans, serum samples were collected from 16 patients infected with SARS-CoV-2 and 20 healthy donors. The binding of the serum antibody to RBD was detected with ELISA as described above. In brief, recombinant RBD protein was used to coat 96-well microtitre plates. After blocking with 1\% BSA, 1:5 diluted serum was added and the plates were incubated for $1 \mathrm{~h}$ at room temperature, followed by four washes with PBST. Bound antibodies were detected with HRP-conjugated antibodies (anti-human IgG/IgM antibody) at a dilution of $1 / 2,000$. For the detection of IgM, serum samples were added to IgG sorbents and the supernatant was collected for further detection by centrifugation. SARS-CoV-2 infections were confirmed by RT-qPCR for all patients with COVID-19 using a 2019-nCoV nucleic acid detection kit. The use of this case series and healthy donors was approved by the institutional ethics board of Sichuan Provincial People's Hospital. Informed consent was obtained from all participants.

To investigate cell-mediated immune responses, mice immunized with the RBD of the S protein or PBS were euthanized to isolate lymphocytes. Lymphocytes were used to analyse IL-4 and IFN $\gamma$ expression in an ELISA assay. In brief, lymphocytes isolated from the spleens of immunized or PBS-treated mice were cultured in RPMI 1640 medium supplied with $10 \%$ (v/v) FBS, $100 \mathrm{U} / \mathrm{ml}$ penicillin, $100 \mu \mathrm{g} / \mathrm{ml}$ streptomycin, $1 \mathrm{mM}$ pyruvate (all from Gibco), $50 \mu \mathrm{M} \beta$-mercaptoethanol and $20 \mathrm{U} / \mathrm{ml}$ IL-2 (all from Sigma-Aldrich). Simultaneously, $1 \mu \mathrm{g} / \mathrm{ml}$ RBD protein was added to activate cells. These cells $\left(1 \times 10^{6}\right.$ per well) were incubated for $72 \mathrm{~h}$ at $37^{\circ} \mathrm{C}$. Cells cultured without RBD protein were used as negative control. The supernatants were collected for ELISA assays to analyse cytokine levels. Potential memory lymphocytes against the recombinant RBD protein were identified by analysing the phenotypes of these cultured lymphocytes by flow cytometry (see 'Flow cytometry').

\section{Ethics approval}

We obtained approval from the institutional ethics board of Sichuan Provincial People's Hospital. Data collection and analysis of patients were also required by the National Health Commission of the People's Republic of China to be part of a continuing public health outbreak investigation.

\section{Measurement of the inhibition of RBD binding to cell-surface ACE2}

A binding assay of RBD-Fc with ACE2 was performed by flow cytometry as previously described ${ }^{28}$. In brief, ACE2 ${ }^{+}$Huh7 cells (a human hepatoma cell line) were collected and washed with Hanks' balanced salt solution (HBSS). The recombinant SARS-CoV-2 RBD-Fc fusion protein was added to the cells at a final concentration of $0.1 \mu \mathrm{g} / \mathrm{ml}$ in the presence or absence of sera (sera from mice immunized with the RBD vaccine on day 7 after the first vaccination and sera from mice treated with PBS as a control) at different dilutions. The cells were incubated at room temperature for $30 \mathrm{~min}$. Cells were washed three times with HBSS and then stained with an anti-human IgG-FITC conjugate (Sigma-Aldrich) at 1:50 dilution for an additional $30 \mathrm{~min}$. After washing, the cells were fixed with $1 \%$ formaldehyde in PBS and processed using the NovoCyte 
Flow Cytometer (ACEA Biosciences). Results were analysed with FlowJo V10 software.

\section{Neutralization of live SARS-CoV-2 and pseudovirus}

To assess the neutralization of the SARS-CoV-2 infection, Vero E6 cells $\left(5 \times 10^{4}\right)$ were seeded in 96 -well plates and grown overnight. SARS-CoV-2 at the $50 \%$ tissue-culture infectious dose $\left(\mathrm{TCID}_{50}\right)$ was preincubated with an equal volume of diluted sera from immunized mice or monkeys before addition to cells. After incubation at $37^{\circ} \mathrm{C}$ for $1 \mathrm{~h}$, the mixture was added to Vero E6 cells. On day 3 after infection, cytopathogenic effects were recorded under the microscope and the neutralizing titres of the dilutions of sera that resulted in complete or $\mathrm{EC}_{50}$ inhibition were calculated $^{28}$.

A neutralization assay using the pseudovirus was performed by measuring the infection of ACE2-transfected HEK293T cells as previously described ${ }^{13,16,29}$. In brief, HEK293T cells were transfected with a plasmid encoding codon-optimized SARS-CoV-2 S protein, a green fluorescent protein (GFP) vector (pLenti-eGFP) and a gag/pol expression plasmid using polyetherimide (Sigma-Aldrich, 700193). After transfection for $6 \mathrm{~h}$, the medium was replaced with new complete culture medium. Then $48 \mathrm{~h}$ after transfection, the culture supernatants containing eGFP-expressing pseudoviruses were collected, filtered through a $0.45-\mu \mathrm{m}$ pore-size filter (Millipore, SLHP033RB), concentrated with an ultrafiltration centrifugal tube and stored in $1 \mathrm{ml}$ at aliquots at $-80^{\circ} \mathrm{C}$ until use. eGFP-expressing pseudotype viruses were produced by co-transfecting HEK293T cells with a plasmid encoding codon-optimized SARS-CoV-2 S protein, a pLenti-EGFP vector and a gag/pol expression plasmid. Supernatants containing the pseudovirus were collected $48 \mathrm{~h}$ after transfection and preincubated with sera from the immunized mice, rabbits, or monkeys at various dilutions or with control sera. After incubation for $1 \mathrm{~h}$ at $37^{\circ} \mathrm{C}$, the mixture was added to ACE2-transfected HEK293T cells to detect viral infectivity. The medium was changed the following day and $48 \mathrm{~h}$ after infection, eGFP expression in infected cells was determined by fluorescent microscopy and flow cytometry.

The 50\% neutralization activities in ACE2-transfected HEK293T cells was measured using a SARS-CoV-2 pseudovirus coated with the S protein. This pseudovirus was generated based on the backbone of VSV G pseudotype virus (G* $\mathrm{G}^{*}-\mathrm{VSV}$ ) as described ${ }^{30}$. In brief, pcDNA3.1.S2 that expresses SARS-CoV-2S was transfected into HEK293T cells using Lipofectamine 3000 (Invitrogen, L3000015). Then, $24 \mathrm{~h}$ after transfection, HEK293T cells were infected with $G^{*} \Delta G$-VSV using a multiplicity of infection of four. After $2 \mathrm{~h}$, transfected cells were washed with PBS, fresh culture medium was added and the cells were incubated for $24 \mathrm{~h}$. The culture supernatants containing pseudoviruses were collected, filtered through $0.45-\mu \mathrm{m}$ pore-size filters (Millipore, SLHP033RB) and stored at $-80^{\circ} \mathrm{C}$ until use. Infection of HEK 293 cells that express human ACE2 by the SARS-CoV-2 pseudovirus was determined in the presence of serum from mice, rabbits or monkeys in a threefold dilution series. The backbone of the SARS-CoV-2 pseudovirus was provided by VSV G pseudotype virus (G* $\Delta \mathrm{G}-\mathrm{VSV})$, which contains expression cassettes for firefly luciferase instead of VSV-G in the VSV genome ${ }^{30}$.

\section{Challenge of non-human primates (M. mulatta) with live SARS-CoV-2}

All procedures involving non-human primates were reviewed and approved by the Institutional Animal Care and Use Committee of Institute of Medical Biology, Chinese Academy of Medical Sciences, and performed in the ABSL-4 facility of Kunming National High-level Biosafety Primate Research Center. Twelve adult non-human primates (5-9 years old) were used for the challenge study with live SARS-CoV-2. Animals were assigned to the following groups: (1) immunization with $40 \mu \mathrm{g}$ RBD protein with aluminium hydroxide adjuvant per dose $(n=4)$; (2) immunization with $20 \mu \mathrm{g}$ RBD protein with aluminium hydroxide per dose $(n=3)$; (3) treatment with PBS (control treatment, $n=3$ ); and (4) treatment with the aluminium hydroxide adjuvant alone $(n=2)$. Non-human primates were immunized with two injections on days 0 and 7 by intramuscular injection and then challenged with SARS-CoV-2 intranasally $\left(0.5 \mathrm{ml}, 10^{6} \mathrm{PFU} / \mathrm{ml}\right)$ on day 28 after the first vaccination. A RT-qPCR assay was used to measure viral gRNA and sgRNA (the latter of which is indicative of virus replication). The viral loads in lung tissues, throat swabs and anal swabs were measured by RT-qPCR. The primer and probe sequences used were derived from the $N$ gene (forward, $5^{\prime}$-GGGGAACTTCTCCTGCTAGAAT- $3^{\prime}$; reverse, 5'-CAGACATTTTGCTCTCAAGCTG-3'; probe, 5'-FAM-TTGCTGCTG CTTGACAGATT-TAMRA-3'), according to the sequences recommended by WHO and China CDC. SARS-CoV-2 $E$ gene subgenomic mRNA, indicative of virus replication, was assessed by RT-qPCR as previously described ${ }^{31}$, using the following primer and probe sequences (forward, 5'-CGATCTCTTGTAGATCTGTTCTC-3'; reverse, 5'-ATATTGCAGCAGTACGCACACA-3'; probe, 5'-FAM-CGAAGCGCAGTAA GGATGGCTAGTGT-Quencher-3').

For paraffin-embedded sections, tissues were collected, fixed in $10 \%$ neutral-buffered formalin and embedded in paraffin, and $5-\mu \mathrm{m}$ sections were prepared for standard haematoxylin and eosin staining.

\section{Adoptive therapy of splenic $\mathrm{T}$ cells or immune sera in the mice challenged with live SARS-CoV-2}

All procedures associated with the mouse study were reviewed and approved by the Institutional Animal Use and Care Committee of the Institute of Laboratory Animal Science, Peking Union Medical College. Mouse studies were performed in an animal biosafety level 3 facility using HEPA-filtered isolators. Experiments in which mice were infected with SARS-CoV-2 were performed using specific pathogen-free transgenic hACE2 mice, for which a colony was established by the Institute of Laboratory Animal Science, Peking Union Medical College. Transgenic mice were generated by microinjecting a transgene comprising the mouse ACE2 promoter driving the human ACE2 coding sequence into the pronuclei of fertilized ova from ICR mice, as described in detail elsewhere $^{32}$. The transgenic hACE2 mice with C57BL/6 background ${ }^{33}$ were provided by the National Institutes for Food and Drug Control.

Adoptive therapy of splenic T cells was conducted as previously described $^{23,34}$. hACE2 mice with C57BL/6 background received $5 \times 10^{7}$ splenic $\mathrm{T}$ cells isolated either from mice with the same $\mathrm{C} 57 \mathrm{BL} / 6$ background 9 days after the third dose of the candidate vaccine or from mice treated with PBS as a control.

Adoptive therapy using sera has previously been described ${ }^{26}$. Adoptive therapy using immune sera was performed using $0.1 \mathrm{ml}$ of pooled sera from immunized mice. In addition, hACE2 mice with ICR background received $0.8 \mathrm{ml}$ sera from mice that were immunized for 7 days with a single dose of the vaccine, and were subsequently challenged with live SARS-CoV-2. The mice were euthanized 5 days after the challenge with live virus. The viral load in lung tissues, lung histopathological changes and changes in body weight were subsequently evaluated. Viral loads in the lung tissues were measured by RT-qPCR. Sections of lung tissues were stained by haematoxylin and eosin and evaluated using light microscopy.

\section{Flow cytometry}

T cells were evaluated with flow cytometry as previously described ${ }^{35}$. Mice immunized with the RBD of the S protein or PBS were euthanized and the lymphocytes were collected. The lymphocytes were cultured in RPMI 1640 medium supplied with $10 \%$ (v/v) FBS, $100 \mathrm{U} / \mathrm{ml}$ penicillin, $100 \mu \mathrm{g} / \mathrm{ml}$ streptomycin, $1 \mathrm{mM}$ pyruvate (all from Gibco), $50 \mu \mathrm{M}$ $\beta$-mercaptoethanol, $20 \mathrm{U} / \mathrm{ml} \mathrm{IL-2} \mathrm{(all} \mathrm{from} \mathrm{Sigma-Aldrich)} \mathrm{for} 72 \mathrm{~h}$. At the same time, $1 \mu \mathrm{g} / \mathrm{ml}$ RBD of the $S$ protein was added to activate cells. Brefeldin A (BD Biosciences) was administered 4-6 h before staining to block intracellular cytokine secretion. Cells were then washed in PBS (Gibco) and stained for $30 \mathrm{~min}$ at $4{ }^{\circ} \mathrm{C}$ with anti-CD8, anti-CD4, anti-CD44, anti-B220 and anti-MHC-II antibodies (all from 
BioLegend). Afterwards, cells were fixed and permeabilized to facilitate intracellular staining with anti-IFN $\gamma$ and anti-IL-4 antibodies (all from BioLegend). Flow cytometry data were acquired on a NovoCyte Flow Cytometer (ACEA Biosciences) and analysed using FlowJo V10 software.

\section{Statistical analysis}

Statistical analyses were performed using Prism 8.0 (GraphPad software). Comparisons among multiple groups across multiple time points were performed using a two-way ANOVA with Tukey's multiple comparison post hoc test. Comparisons among multiple groups were performed using a one-way ANOVA followed by Tukey's multiple comparison post hoc test. Comparisons between two groups were performed using unpaired Student's $t$-tests. $P$ values of $<0.05$ were considered significant. ${ }^{*} P<0.05,{ }^{* *} P<0.01,{ }^{* * *} P<0.001,{ }^{* * * *} P<0.0001$. NS, not significant.

\section{Reporting summary}

Further information on research design is available in the Nature Research Reporting Summary linked to this paper.

\section{Data availability}

Reference SARS-CoV-2 RNA and protein sequences were downloaded from the NCBI database. All data are available in the paper or the Supplementary Information.

23. Wang, Y. \& Tian, Z. New energy setup strategy for intact N-glycopeptides characterization using higher-energy collisional dissociation. J. Am. Soc. Mass Spectrom. 31, 651-657 (2020).

24. Liu, M.-Q. et al. pGlyco 2.0 enables precision N-glycoproteomics with comprehensive quality control and one-step mass spectrometry for intact glycopeptide identification. Nat. Commun. 8, 438 (2017)

25. Yang, W., Ao, M., Hu, Y., Li, Q. K. \& Zhang, H. Mapping the O-glycoproteome using site-specific extraction of O-linked glycopeptides (EXoO). Mol. Syst. Biol. 14, e8486 (2018).

26. Gupta, M., Mahanty, S., Bray, M., Ahmed, R. \& Rollin, P. E. Passive transfer of antibodies protects immunocompetent and imunodeficient mice against lethal Ebola virus infection without complete inhibition of viral replication. J. Virol. 75, 4649-4654 (2001).

27. Stadlmann, J. et al. Comparative glycoproteomics of stem cells identifies new players in ricin toxicity. Nature 549, 538-542 (2017).

28. He, Y. et al. Receptor-binding domain of SARS-CoV spike protein induces highly potent neutralizing antibodies: implication for developing subunit vaccine. Biochem. Biophys. Res. Commun. 324, 773-781 (2004)

29. He, Y., Lu, H., Siddiqui, P., Zhou, Y. \& Jiang, S. Receptor-binding domain of severe acute respiratory syndrome coronavirus spike protein contains multiple conformation-dependent epitopes that induce highly potent neutralizing antibodies. J. Immunol. 174, 4908-4915 (2005)
30. Nie, J. et al. Establishment and validation of a pseudovirus neutralization assay for SARS-CoV-2. Emerg. Microbes Infect. 9, 680-686 (2020).

31. Wölfel, R. et al. Virological assessment of hospitalized patients with COVID-2019. Nature 581, 465-469 (2020)

32. Bao, L. et al. The pathogenicity of SARS-CoV-2 in hACE2 transgenic mice. Nature $\mathbf{5 8 3}$ $830-833(2020)$

33. Sun, S. H. et al. A mouse model of SARS-CoV-2 infection and pathogenesis. Cell Host Microbe 28, 124-133 (2020).

34. Zhao, J., Zhao, J. \& Perlman, S. T cell responses are required for protection from clinical disease and for virus clearance in severe acute respiratory syndrome coronavirus-infected mice. J. Virol. 84, 9318-9325 (2010).

35. Xiao, Y. et al. Adsorption of recombinant poxvirus L1-protein to aluminum hydroxide/CpG vaccine adjuvants enhances immune responses and protection of mice from vaccinia virus challenge. Vaccine 31, 319-326 (2013).

Acknowledgements We thank Z. Li, G. Wei, Q. Chen, H. Yan, X. Zhou, X. Wu, F. Fu, H. Yang and $X$. Wu for assisting with the maintenance of the mouse facility, the collection of sera, and providing cell culture, ELISA assays and other technical support. This work was supported by the National Key Research and Development Program of China (2016YFA0201402). National Natural Science Foundation Regional Innovation and Development (U19A2003), the National Major Scientific and Technological Special Project for 'Significant New Drugs Development' (2018ZX09733001), the projects for the vaccine by the Ministry of Science and Technology, National Health Commission, Ministry of Education of the People's Republic of China, Sichuan province, National Key Research and Development Project of China (2016YFD0500304). The special research fund on COVID-19 of Sichuan province (2020YFS0010), the special research fund on COVID-19 of West China Hospital Sichuan University (HX-2019-nCoV-004) and Macao Science and Technology Development Fund (FDCT).

Author contributions X.W., G. Lu, K.Z. and Y. Wei conceived and supervised the research, and designed the experiments. Z.C., F. Yang, F. Ye, S. Lin, H.C., Y. Zheng, L.Y., J. Li, Y. Huang and D.L. performed gene cloning, expression and protein purification. Y.G., H.D., Z.W., Jinliang Yang, Z.T., L.D., W.C. and Y.P. performed LC-MS/MS analysis to identify glycosylation sites. C.Q., L.B., H.G., W.D., Y.X., Q.L., F.L., S.W., Guanpeng Wang, P.Y. and Y.Q. performed the mouse challenge experiments using live SARS-CoV-2. A.T., W. Huang, Y. Wang and Y.L. performed the neutralization of pseudovirus infection experiments and measurements of the inhibition of the binding of the RBD of S to cell-expressed ACE2. Jingyun Yang, X.W., W.W., Z.B., F.M., M.L., G.S. Z. Zhao, X.C., C.F. and Z.Q. performed the vaccine formulation and vaccinations in mice. X.C., X.P., S. Lu, H. Liu, YY. Y. Zhao, WY, MY, J.X, JW. H. Li, H.W., D.K., Z.H., W.G., Y. He, X.S., C.C., Z.X., S.Y. and L.C. performed the vaccine formulation and vaccinations in rabbits and monkeys, X.W., Jingyun Yang, Z.B., F.M., M.L., S.C., G.S., Z.Y., F.-S.W. and M.S. performed identified serum antibodies against the RBD of S. Jinliang Yang and X.J. performed SPR analysis. H. Lei, X.L. and Z. Zhang performed flow cytometry. X.W. Jingyun Yang, W.W. M. Wang, W.H. S.C. C.H., J. Luo, P.L., J.Y.-N.L., X.M., G. Li, M.G., Guangyu Wang and K.Z. analysed and interpreted the data. J.Y.-N.L. and K.Z. assisted with the adjustments of directions and interpretation of the mechanistic aspects of the results. X.W., J.Y.-N.L., K.Z., G. Lu, and M. Wu wrote the manuscript.

Competing interests G.Lu, J. Li, J.Y.-N.L., G.S., L.Y., W.Y., Jinliang Yang, X.W., Z.W., W.W., Y.Wei, K.Z. and Z. Zhao filed patents on vaccine technologies.

Additional information

Supplementary information is available for this paper at https://doi.org/10.1038/s41586-0202599-8.

Correspondence and requests for materials should be addressed to K.Z., G.L. or X.W. Peer review information Nature thanks Wolfgang Baumgärtner and the other, anonymous, reviewer(s) for their contribution to the peer review of this work. Reprints and permissions information is available at http://www.nature.com/reprints. 

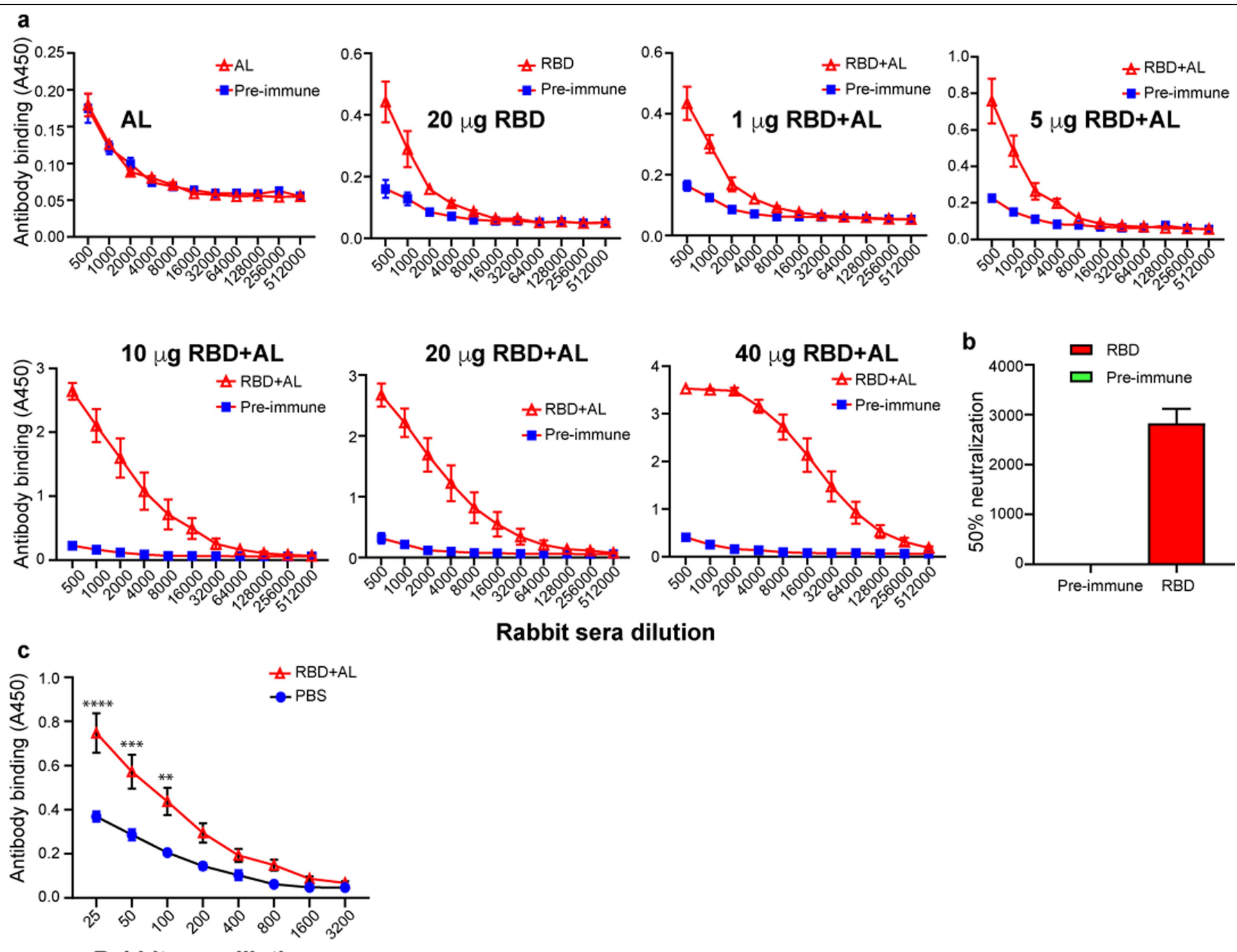

Rabbit sera dilution

\section{Rabbit sera dilution}

Extended Data Fig. 1 | Identification of serum antibodies against the RBD of the $S$ protein and neutralizing antibodies against SARS-CoV-2 pseudovirus in rabbits. a, The rabbits were immunized with $1-40 \mu \mathrm{g}$ recombinant $\mathrm{RBD}$ protein per rabbit in $500 \mu \mathrm{l}$ in the presence of aluminium hydroxide, or with only aluminium hydroxide or PBS. Rabbit were immunized with three vaccinations on days 0,14 and 21 , and sera were collected 7 days after each boost. Sera collected from the rabbits 7 days after the third vaccination were tested at different dilutions for IgGs against the RBD of the S protein using ELISA. Data are mean \pm s.e.m. of the sera of $n=6$ rabbits per group, except for the aluminium hydroxide group, for which $n=4$. AL, aluminium hydroxide; $\mathrm{RBD}+\mathrm{AL}$, the addition of the recombinant RBD protein to aluminium hydroxide. b, Potent neutralization of the SARS-CoV-2 pseudovirus infection by sera from rabbits immunized with the recombinant $R B D$ vaccine. Infection of HEK293 cells expressing human ACE2 by SARS-CoV-2 pseudovirus was determined in the presence of rabbit sera in a threefold dilution series. The percentage of neutralization is shown as mean \pm s.e.m. The sera from the rabbits immunized 7 days after the third vaccination with the dose of $20 \mu \mathrm{g}$ recombinant RBD protein per rabbit in $500 \mu$ in the presence of aluminium hydroxide is the same as described in $\mathbf{a}$. The neutralization assay of the SARS-CoV-2 pseudovirus was performed as described in the Methods. c, The rabbits were immunized in the same way as a. Sera were collected from the rabbits 7 days after the first vaccination with a dose of $40 \mu \mathrm{g}$ recombinant RBD protein and were tested at different dilutions for IgMs against the RBD of the $S$ protein using ELISA. 

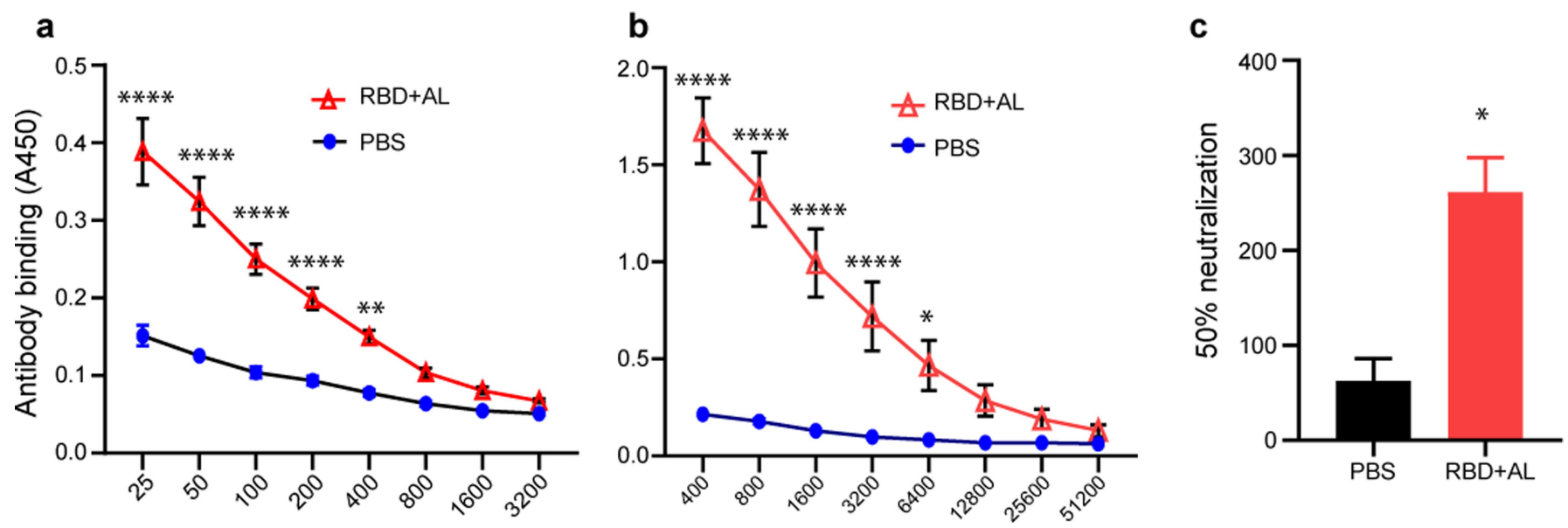

\section{d}

\section{Sera dilution}

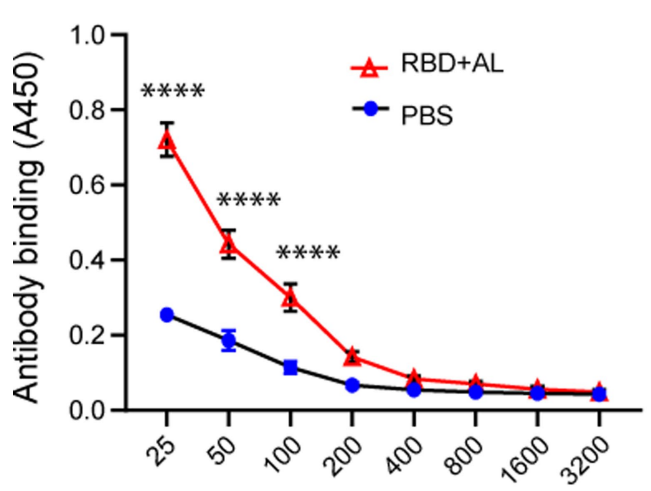

Sera dilution

Extended Data Fig. 2 | Identification of serum antibodies against the RBD of the $S$ protein and immunization of non-human primates. $a, b$, The non-human primates ( $M$. mulatta) were immunized with $40 \mu$ g recombinant RBD protein (in $1 \mathrm{ml}$ ) per monkey in the presence of aluminium hydroxide on day 0 and day 7 , and sera were obtained at 7 days (a) and 14 days (b) after the first vaccination or before the vaccination (Pre-immune). Monkeys treated with PBS were used as a control. Sera were tested at different dilutions for IgGs against the recombinant RBD protein using ELISA as described in the Methods. Data are mean \pm s.e.m. of the sera from $n=10$ monkeys per group. $P$ values were determined by two-way ANOVA and are shown for the differences in IgG levels between the RBD + AL and PBS groups. c, The neutralization of the infection with the SARS-CoV-2 pseudovirus by sera from non-human primates. The neutralization assays were performed with sera from monkeys 14 days after the first vaccination as in $\mathbf{b}$. $\mathrm{EC}_{50}$ data are mean \pm s.e.m. The neutralization assay of the SARS-CoV-2 pseudovirus is described in the Methods. d, Sera were collected from the monkeys 7 days after the first vaccination (as described in a) with the dose of $40 \mu \mathrm{g}$ recombinant RBD protein and were tested at different dilution for IgMs against the RBD of the S protein using ELISA. 

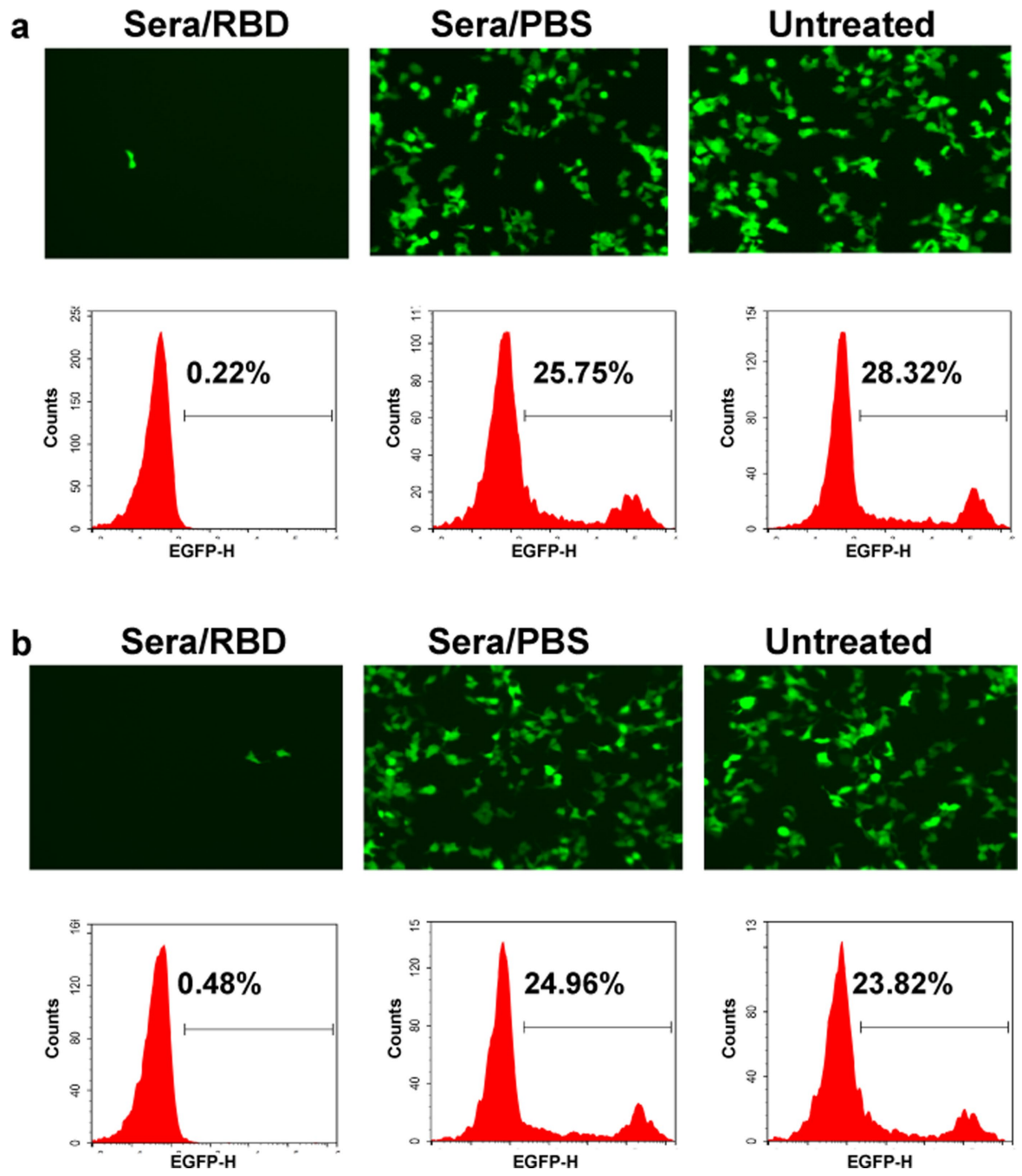

Extended Data Fig. 3 | The neutralization of the SARS-CoV-2 pseudovirus infection by the sera from mice or rabbits. a, Supernatants containing the SARS-CoV-2 pseudovirus were preincubated with sera from mice that were twofold serially diluted. After incubation for $1 \mathrm{~h}$ at $37^{\circ} \mathrm{C}$, the mixture was added to ACE2-transfected HEK293T cells to detect viral infectivity. The number of GFP-positive cells was determined by fluorescent microscopy and flow cytometry. Sera/RBD, sera pooled from 5 mice immunized with the RBD vaccine collected on day 14 after the first vaccination; Sera/PBS, sera from mice treated with PBS as a control; Untreated, infection with SARS-CoV-2

pseudovirus without serum treatment. b. The neutralization of the infection of SARS-CoV-2 pseudovirus was performed using the sera from rabbits 14 days after the first vaccination in the same way as described in $\mathbf{a}$. 
a

Untreated
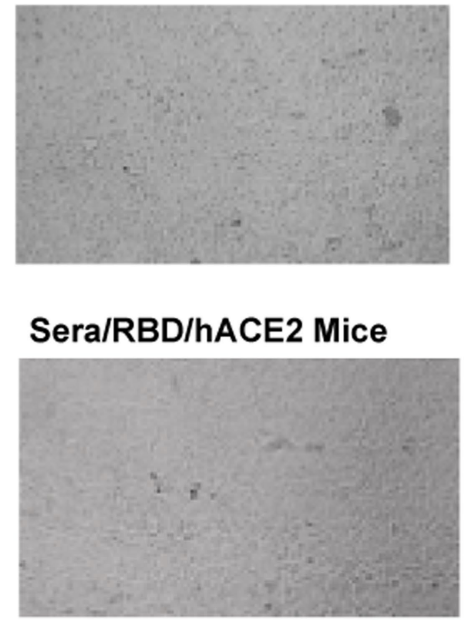

Sera/PBS/hACE2 Mice

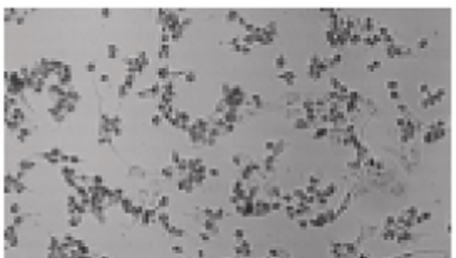

Extended Data Fig. 4 | The induction of the neutralizing antibodies against live SARS-CoV-2 in transgenic hACE2 and wild-type mice. a, Transgenic hACE2 and wild-type mice were immunized with $10 \mu \mathrm{g}$ recombinant RBD protein per mouse in $50 \mu \mathrm{l}$ in the presence of aluminium hydroxide or treated with PBS alone. Sera were collected from the mice 14 days after the second vaccination. To assess the neutralization of the SARS-CoV-2 infection, Vero E6 cells $\left(5 \times 10^{4}\right)$ were seeded in 96 -well plates and grown overnight. SARS-CoV-2 b

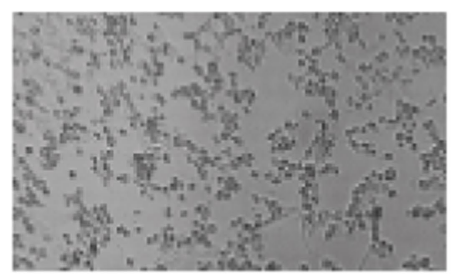

\section{Sera/RBD/Wild-type Mice}
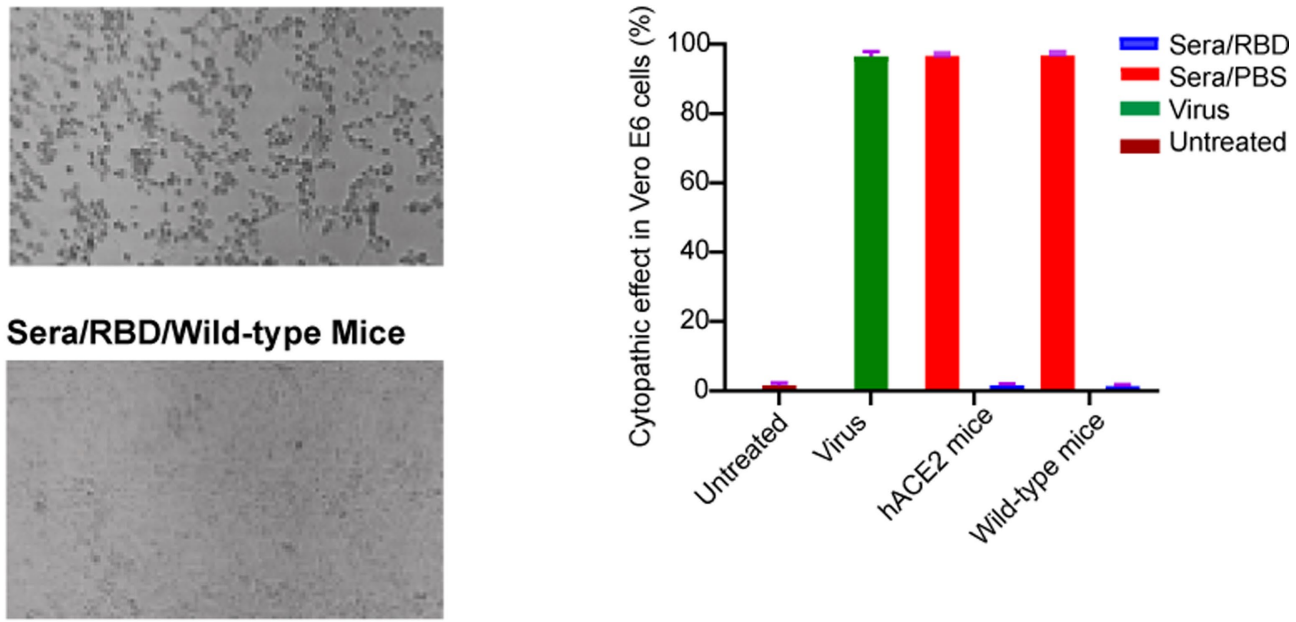

$\left(100 \mathrm{TCID}_{50}\right)$ was preincubated with an equal volume of diluted sera before addition to the cells. After incubation at $37^{\circ} \mathrm{C}$ for $1 \mathrm{~h}$, the mixture was added to Vero 6 cells. The cytopathogenic effects were recorded under the microscope and the neutralizing titres of the dilutions of sera that resulted in complete inhibition were calculated. $b$, The percentages of cells with cytopathogenic effects were calculated. Data are mean \pm s.d. 
a

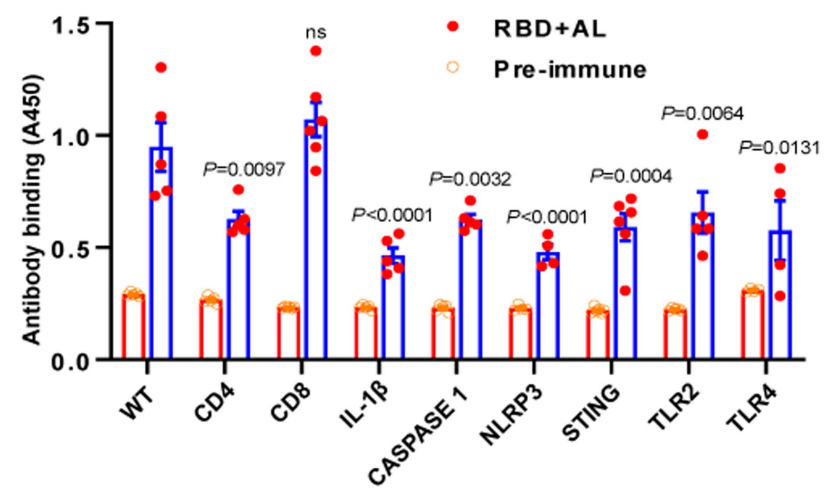

b
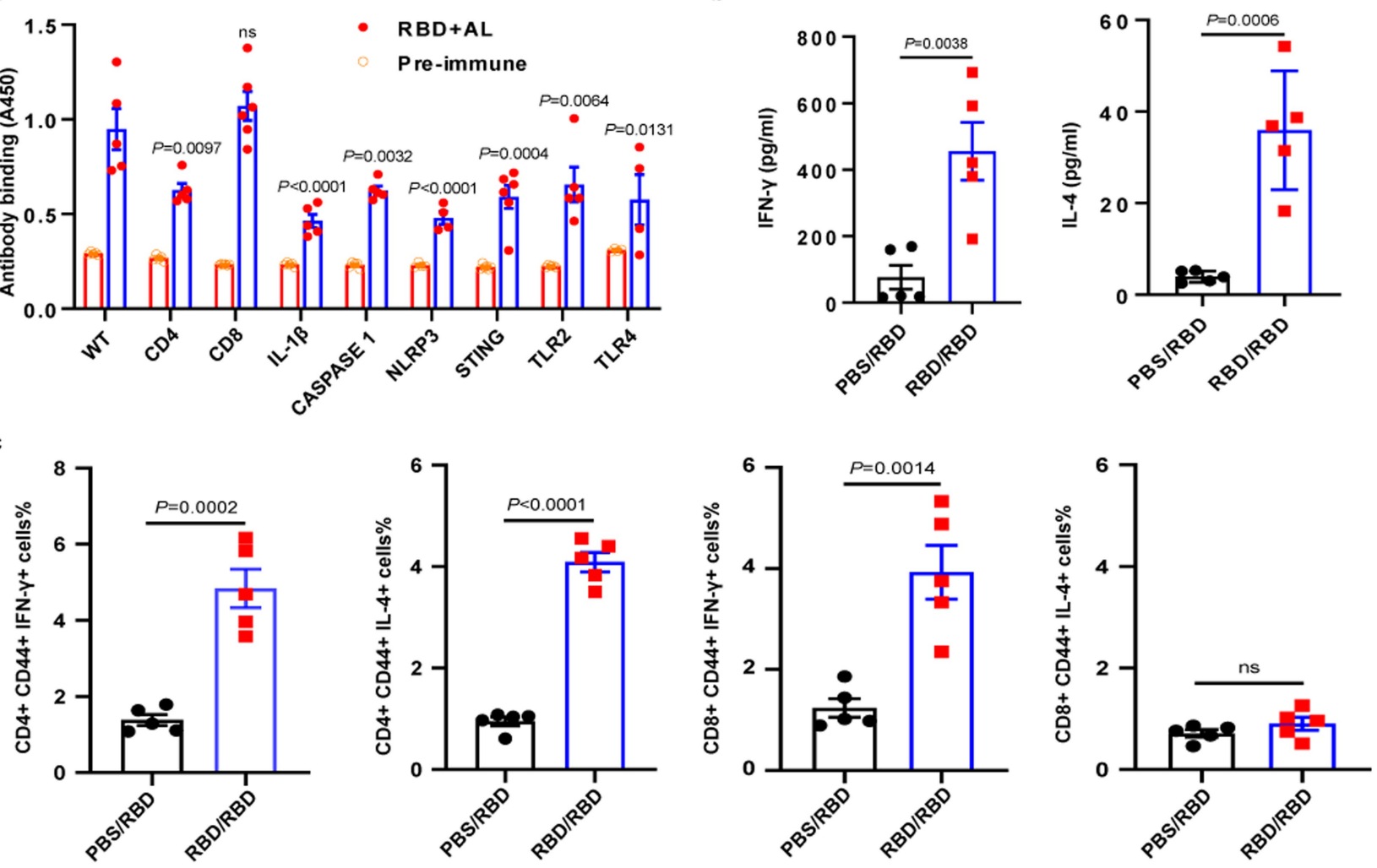

d

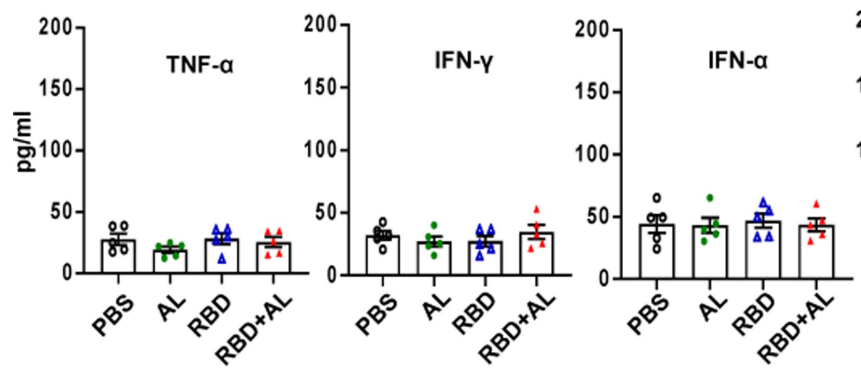

Extended Data Fig. 5 | Pathways stimulated by the recombinant RBD and involved in the immune response. a, Wild-type $\mathrm{C} 57 \mathrm{BL} / 6 \mathrm{mice}$ and $\mathrm{Cd4^{-/ }}$,

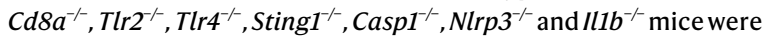
immunized with the recombinant RBD protein ( $5 \mu \mathrm{g}$ per mouse) and sera were collected on day 7 after the first dose of the vaccine and were tested for antibodies against the RBD at a dilution of 1:25. Data are mean \pm s.e.m. $P$ values were determined by two-way ANOVA (4-6 mice per group). Similar results were obtained in two independent experiments. b. Cytokines produced by the spleen lymphocytes were detected by ELISA after stimulation with the recombinant RBD. Mice immunized with the candidate RBD vaccine or treated with PBS were euthanized 7 days after the first dose of vaccine to isolate lymphocytes, which were then stimulated with recombinant RBD for 3 days.

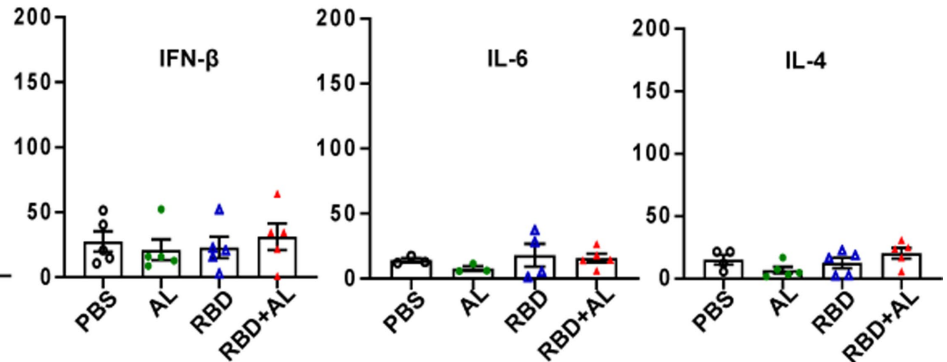

Subsequently, the supernatants were collected to assess the levels of secreted IL- 4 and IFN $\gamma$ by ELISA assays, as described in the Methods. Data are mean \pm s.d. $P$ values were determined by unpaired Student's $t$-tests (5 mice per group). Similar results were obtained in three independent experiments. $c$, The lymphocytes in the spleen were collected from mice treated with the recombinant $\mathrm{RBD}(5 \mu \mathrm{g}$ per mouse) 7 days after the first vaccination and were incubated with RBD for 3 days. The number of RBD-reactive memory CD4 or CD8 T cells was analysed by flow cy tometry by gating on CD $44^{\text {high }} \mathrm{B} 220^{-} \mathrm{MHC}-\mathrm{II}^{-}$ $\mathrm{CD}^{+}$or $\mathrm{CD}^{+} \mathrm{T}$ cells that were IFN $\gamma^{+}$or $\mathrm{IL}_{-} 4^{+}$, as described in the Methods. Similar results were obtained in three independent experiments. d, Sera were collected from mice 7 days after the first dose of vaccine and plasma level of cytokines such as TNF, IFN $\gamma$, IFN $\alpha$, IFN $\beta$, IL-6, IL- 4 were measured by ELISA. 
a

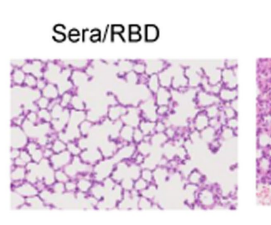

T lymphocytes/RBD

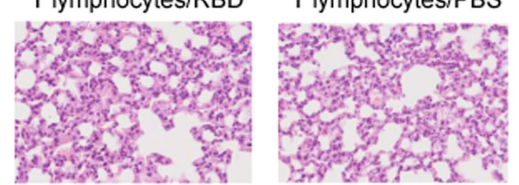

b

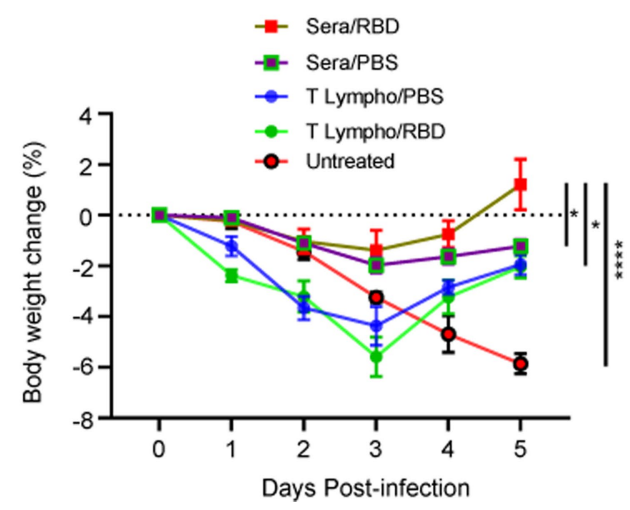

Extended Data Fig. 6| Adoptive therapy of immune sera from vaccinated mice. a, b, hACE2 mice with $\mathrm{C} 57 \mathrm{BL} / 6$ background received $0.1 \mathrm{ml}$ of pooled sera or $5 \times 10^{7}$ splenic T cells isolated from mice with the same $\mathrm{C} 57 \mathrm{BL} / 6$ background 9 days after the third dose of the candidate vaccine or from mice treated with PBS as a control. The mice were euthanized 5 days after the challenge with live virus, and viral replication in lung tissues (Fig. 4a), lung histopathological changes (a) and bodyweight changes (b) were evaluated. hACE2 mice with ICR background received $0.8 \mathrm{ml}$ sera from immunized mice (immunized mice c

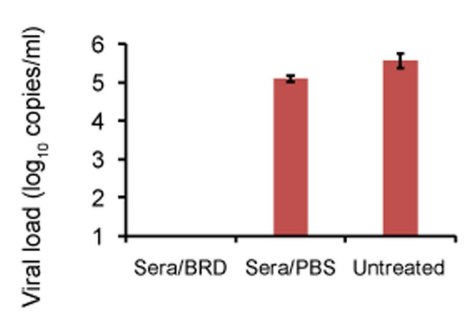

d

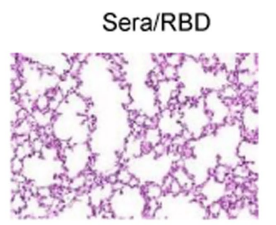
Sera/PBS

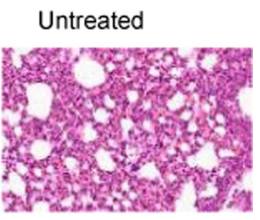

e

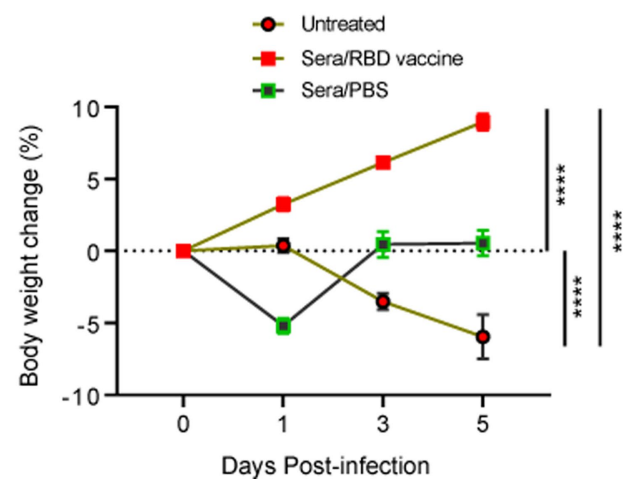

received a single dose of the vaccine and sera were collected after 7 days). The hACE2 mice with ICR background were then challenged with live SARS-CoV-2. The mice were euthanized 5 days after the challenge with the live virus, and viral replication in lung tissues (c), lung histopathological changes $(\mathbf{d})$, and body weight changes (e) were evaluated. Adoptive therapy was performed as described in the Methods. Sera/RBD, sera pooled from the mice immunized with the RBD vaccine; Sera/PBS, sera from mice treated with PBS as a control; Untreated, infection with live SARS-CoV-2 without sera. 


\section{Reporting Summary}

Nature Research wishes to improve the reproducibility of the work that we publish. This form provides structure for consistency and transparency in reporting. For further information on Nature Research policies, see Authors \& Referees and the Editorial Policy Checklist.

\section{Statistics}

For all statistical analyses, confirm that the following items are present in the figure legend, table legend, main text, or Methods section.

n/a Confirmed

$\square \searrow$ The exact sample size $(n)$ for each experimental group/condition, given as a discrete number and unit of measurement

$\square$ \A statement on whether measurements were taken from distinct samples or whether the same sample was measured repeatedly

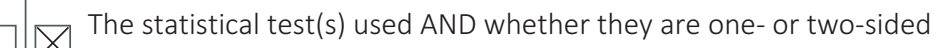

$\triangle$ Only common tests should be described solely by name; describe more complex techniques in the Methods section.

$\square$ A description of all covariates tested

$\square \bigotimes$ A description of any assumptions or corrections, such as tests of normality and adjustment for multiple comparisons

$\square$ A full description of the statistical parameters including central tendency (e.g. means) or other basic estimates (e.g. regression coefficient)

$\bigotimes$ AND variation (e.g. standard deviation) or associated estimates of uncertainty (e.g. confidence intervals)

$\bigotimes$ For null hypothesis testing, the test statistic (e.g. $F, t, r$ ) with confidence intervals, effect sizes, degrees of freedom and $P$ value noted

Give P values as exact values whenever suitable.

Х $\square$ For Bayesian analysis, information on the choice of priors and Markov chain Monte Carlo settings

Х $\square$ For hierarchical and complex designs, identification of the appropriate level for tests and full reporting of outcomes

$\bigotimes \square$ Estimates of effect sizes (e.g. Cohen's $d$, Pearson's $r$ ), indicating how they were calculated

Our web collection on statistics for biologists contains articles on many of the points above.

\section{Software and code}

Policy information about availability of computer code

Data collection 1. Affinity and kinetics data for RBD were collected using Biacore 8K by GE Healthcare (General Electric Company).

2. Flow cytometric data were collected using LSRFortessa (BD Biosciences) or NovoCyte (ACEA bioscience. Inc).

3. Pathologic slides were digitized using Olympus IX73 microscope (Olympus Corporation)

using 20x/0.8NA objective and filters for A488.

4. Western blot data were collected using Clinx ChemiScope Series (Clinx Science Instrument Co., Ltd).

5. Enzyme linked Immunosorbent Assay data were collected using CYTATION 3 Imaging Reader (BioTek Instruments, Inc. ).

Data analysis CellSens Standard software

NovoExpress 1.4.1

GraphPad Prism version 8.0,

Photoshop(Adobe),

Biacore Insight Evaluation Software,

Gen5 version 3.05,

PyMOL 1.3

For manuscripts utilizing custom algorithms or software that are central to the research but not yet described in published literature, software must be made available to editors/reviewers. We strongly encourage code deposition in a community repository (e.g. GitHub). See the Nature Research guidelines for submitting code \& software for further information. 
Policy information about availability of data

All manuscripts must include a data availability statement. This statement should provide the following information, where applicable:

- Accession codes, unique identifiers, or web links for publicly available datasets

- A list of figures that have associated raw data

- A description of any restrictions on data availability

All reasonable requests will be considered and granted by a data access committee consisted of $Y Q \mathrm{~W}, \mathrm{KZ}, \mathrm{XW} W$

\section{Field-specific reporting}

Please select the one below that is the best fit for your research. If you are not sure, read the appropriate sections before making your selection.

$\bigotimes$ Life sciences $\quad \square$ Behavioural \& social sciences $\quad \square$ Ecological, evolutionary \& environmental sciences

For a reference copy of the document with all sections, see nature.com/documents/nr-reporting-summary-flat.pdf

\section{Life sciences study design}

All studies must disclose on these points even when the disclosure is negative. $\begin{array}{ll}\text { Sample size } & \begin{array}{l}\text { Sample sizes were determined based on our and other investigators experience. No statistical methods were used to predetermine sample } \\ \text { size. }\end{array}\end{array}$

Data exclusions No data were excluded from the analysis.

Replication Most of Experiments were repeated with at least two biologically independent for all results presented in the manuscript.

Randomization Mice of 6- to 10-week old were matched by age and sex and randomly assigned to different treatment groups.

$\begin{array}{lll}\text { Blinding } & \text { Researchers were not blinded in the process of experiments or data analysis since all of the findings are supported by quantitative }\end{array}$ measurement.

\section{Reporting for specific materials, systems and methods}

We require information from authors about some types of materials, experimental systems and methods used in many studies. Here, indicate whether each material, system or method listed is relevant to your study. If you are not sure if a list item applies to your research, read the appropriate section before selecting a response.

\begin{tabular}{l|l}
\multicolumn{3}{l}{ Materials \& experimental sys } \\
\hline $\mathrm{n} / \mathrm{a}$ & Involved in the study \\
\hline & $\bigotimes$ Antibodies \\
$\square$ & $\bigotimes$ Eukaryotic cell lines \\
$\square$ & $\square$ Palaeontology \\
$\square$ & $\bigotimes$ Humals and other organisms \\
$\square$ & $\square$ Clinical data
\end{tabular}

\begin{tabular}{l|l}
\multicolumn{2}{l}{ Methods } \\
\hline n/a & Involved in the study \\
$\square$ & $\square$ ChIP-seq \\
$\square$ & $\bigotimes$ Flow cytometry \\
$\square$ & $\square$ MRI-based neuroimaging
\end{tabular}

\section{Antibodies}

Antibodies used

anti-CD8-FITC, Applicable: FCM, Provide supplier name: BioLegend, Cat: 100706, Clone: 53-6.7, Lot: B278606 anti-CD4-APC, Applicable: FCM, Provide supplier name: BioLegend, Cat: 100412, Clone: GK1.5, Lot: B293107 anti-MHC-II-PE-Cy7, Applicable: FCM, Provide supplier name: BioLegend, Cat: 107630, Clone: M5/114.15.2, Lot: B199459 anti-CD45R-PerCP/Cy5.5, Applicable: FCM, Provide supplier name: BioLegend, Cat: 103236, Clone: RA3-6B2, Lot: B295257 anti-CD44-BV510, Applicable: FCM, Provide supplier name: BioLegend, Cat: 103044, Clone: IM7, Lot: B298812 anti-IFN-ץ-PE, Applicable: FCM, Provide supplier name: BD Bioscience, Cat: 554412, Clone: XMG1.2, Lot: 3217951 anti-IL-4-BV421, Applicable: FCM, Provide supplier name: BioLegend, Cat: 504120, Clone: 11B11, Lot: B282852 rabbit anti-ACE2, Applicable: FCM, Provide supplier name: Sino Biological, Cat: 10108-RP01, Clone: Polyclonal Rabbit IgG, Lot: GR3331742-1

Donkey anti-rabbit IgG-FITC, Applicable: FCM, Provide supplier name: BioLegend, Cat: 406403, Clone: Poly4064, Lot: B228075 anti-His Tag-PE, Applicable: FCM, Provide supplier name: BioLegend, Cat: 362603, Clone: J095G46, Lot: B269138

Mouse IgG-HRP, Applicable: ELISA, Provide supplier name: southern biotech, Cat: 0107-05 
Validation

Antibodies were chosen based on the available literature. Quality of the antibodies used in the study was tested by manufacturer or relevant references cited on the manufacturer's website. Additional information on validation can be found on the manufacturers' websites.

\section{Eukaryotic cell lines}

Policy information about cell lines

Cell line source(s)

HUH-7 cells (JCRB JCRB0403), HEK293T cells (ATCC CRL-11268)

Authentication

Cell lines were authenticated by morphology

Mycoplasma contamination

Neither of the cell lines used in this study tested positive for Mycoplasma.

Commonly misidentified lines (See ICLAC register)

No commonly misidentified lines were used in this study.

\section{Animals and other organisms}

Policy information about studies involving animals; ARRIVE guidelines recommended for reporting animal research

Laboratory animals

Purchased from Jackson Laboratory

B6N.129S2-Casp1tm1Flv/J, (Jax \#016621)

B6.129S6-Cd4tm1Knw/J, (Jax\#002269)

B6.129S2-Cd8atm1Mak/J, (Jax \#002665)

C57BL/6J-Sting1gt/J, (Jax \#17537)

B6.129-TIr2tm1 Kir/J, (Jax \#004650)

B6.B10ScN-T|r4lps-del/JthJ, (Jax \#007227)

B6(Cg)-Ifnar1tm1.2Ees/J, (Jax\#028288)

Purchased from Genentech, Inc., USA

Asc-/-

NIrp3-/-

Provided by Tokyo University of Science

II-1 $\beta-/-$

Purchased from Beijing Vital River Laboratory Animal Technology

CD-1 ${ }^{\circledast}$ (ICR) IGS

$\mathrm{NIH}(\mathrm{S})$

C57BL/6N

Transgenic hACE2 mice with ICR background were provided by the Institute of Laboratory Animal Science, Peking Union Medical College, China.

Transgenic hACE2 mice with C57BL/6 background35 were provided by the National Institutes for Food and Drug Control (NIFDC, Beijing, China)

Rabbits Purchased from Shangdong Benming Biotechnology Limited Company, China, Production License No :

SCXK(Lu)2017-0003.

Mon-human primates (Macaca mulatta) for the immunization and challenge provided by National Kunming High-level Biosafety Primate Research Center, Institute of Medical Biology, Chinese Academy of Medical Sciences and Peking Union Medical College, Yunnan China.

Mon-human primates (Macaca mulatta) for the safety study Purchased from Sichuan Hensu Biotechnology Limited Company, Production License No: SCXK (Chuan) 2019-029.

Wild animals

Field-collected samples

Ethics oversight
No wild animals were used in this study.

No field-collected samples were used in the study.

All animal studies carried out were approved by the Animal Care and Use Committee of Sichuan University (Chengdu, Sichuan, China).

Transgenic hACE2 mice were approved by the Animal Care and Use Committee, the Institute of Laboratory Animal Science, Peking Union Medical College, China.

All procedures involved in the immunization and challenge in the non-human primates study were reviewed and approved by the Institutional Animal Care and Use Committee of Institute of Medical Biology, Chinese Academy of Medical Science, and performed in the ABSL-4 facility of Kunming National High-level Biosafety Primate Research Center, Yunnan, China.

All procedures involved in the immunization in rabbits and safety study in the non-human primates were reviewed and approved by the Institutional Animal Care and Use Committee, National Chengdu Center for Safety Evaluation of Drugs, Chengdu, China. 
Policy information about studies involving human research participants

Population characteristics In order to investigate the potential immunogenicity of S protein RBD as vaccines in human, serum samples were collected from 16 patients infected with SARS-CoV-2 and 20 healthy donors detected with ELISA. All 16 patients with COVID-19 were confirmed by RT-PCR using a 2019-nCoV nucleic acid detection kit. In addition, 20 healthy donors for blood as a control. 16 patients including 12 female, 4 male, age from 23 to 76 years old, common symptoms at onset of illness were fever fatigue, dry cough, myalgia, Chest computed tomographic images show ground glass opacity to varying degree.

Recruitment

This study is not a clinical trial. The blood from 16 patients and 20 healthy donors only for confirming the existence of the antibody reactive to RBD protein of SARS-CoV-2.

Ethics oversight

This case series and healthy donors were approved by the institutional ethics board of Sichuan Provincial People's Hospital

Note that full information on the approval of the study protocol must also be provided in the manuscript.

\section{Flow Cytometry}

Plots

Confirm that:

$\bigotimes$ The axis labels state the marker and fluorochrome used (e.g. CD4-FITC).

$\bigotimes$ The axis scales are clearly visible. Include numbers along axes only for bottom left plot of group (a 'group' is an analysis of identical markers).

\All plots are contour plots with outliers or pseudocolor plots.

$\bigotimes$ A numerical value for number of cells or percentage (with statistics) is provided.

\section{Methodology}

Sample preparation

\begin{abstract}
Splenocytes activation
T cells activation was evaluated with flow cytometry. Mice immunized with S1-RBD or PBS were sacrificed to collect splenocytes. After lysis of red blood cells, splenocytes were cultured in RPMI medium 1640 supplied with 10\% (vol/vol) FBS, $100 \mathrm{U} / \mathrm{ml}$ penicillin, $100 \mu \mathrm{g} / \mathrm{ml}$ streptomycin, $1 \mathrm{mM}$ pyruvate (all from Gibco), $50 \mu \mathrm{M} \beta$-mercaptoethanol (Amresco), and $20 \mathrm{U} / \mathrm{ml} \mathrm{IL}-2$ (Sigma-Aldrich) for $72 \mathrm{~h}$. At the same time, $10 \mu \mathrm{g} / \mathrm{ml}$ S1-RBD was added to activate cells. Brefeldin A (BD Biosciences) was administrated 4-6h before staining to block intracellular cytokine secretion. Cells were then washed in PBS (Biosharp) and stained for $30 \mathrm{~min}$ at $4{ }^{\circ} \mathrm{C}$ with anti-CD8a-FITC (53-6.7), anti-CD4-APC (GK1.5), anti-MHC-II-PE-Cy7 (M5/114.15.2), anti-CD45RPerCP/Cy5.5 (RA3-6B2) and anti-CD44-BV510 (IM7) (all from BioLegend). Afterwards, cells were fixed and permeabilized to facilitate intracellular staining with anti-IFNY-PE (XMG1.2) (BD Bioscience) and anti-IL-4-BV421 (11B11) (BioLegend).
\end{abstract}

\section{ACE2 transfection}

Flow cytometry was utilized to determine efficiency of ACE2 transfection. Two days after transfection, HEK293T cells was harvested and washed with Hanks' balanced salt solution (HBSS, Gibco). Cells were then incubated with polyclonal rabbit antiACE2 (Polyclonal Rabbit IgG) (Sino Biological) in room temperature for 30 min followed by repeated washing with HBSS. Donkey anti-rabbit IgG-FITC (Ploy4064) (BioLegend) was then added to the cell suspension for extra 30 min incubation in room temperature protecting from light. Cells were then fixed with $1 \%$ formaldehyde in PBS.

Inhibition of receptor binding assays

HEK293T cells were transfected with a plasmid encoding human ACE2. At 48 hour post-transfection, HEK293T/ACE2 cells were collected and washed with HBSS. The RBD-His was added to the cells to a final concentration of $1 \mu \mathrm{g} / \mathrm{ml}$ in the presence or absence of the sera at a dilution of 1:5, followed by incubation at room temperature for 30 min. Cells were washed three times with HBSS and then incubated with anti-His Tag-PE (J095G46) (BioLegend) at room temperature for additional 30 min. After washing, cells were fixed with $1 \%$ formaldehyde in PBS.

Challenge of the non-human primates (Macaca mulatta)) with live SARS-CoV-2

Non-human primates were immunized with two injections on day 0, day 7 via the intramuscular route with $20 \mu \mathrm{g}$ or $40 \mu \mathrm{g}$ per dose and then challenged with SARS-CoV-2 intranasally $(0.5 \mathrm{ml}, 106 \mathrm{pfu} / \mathrm{ml})$ on day 28 after the first vaccination. A quantitative real-time reverse transcription-PCR (qRT-PCR) was employed to measure viral genomic RNA (gRNA) and viral subgenomic RNA ( $s g R N A$ ), indicative of virus replication. Viral load in the lung tissues, throat and anal swabs, etc, were measured by qRT-PCR. The primer and probe sequences used were derived from NP gene (Forward: 5'-GGGGAACTTCTCCTGCTAGAAT-3', Reverse: 5'CAGACATTTTGCTCTCAAGCTG-3', Probe : 5'-FAMTTGCTGCTGCTTGACAGATT-TAMRA-3'), according to the sequences recommended by $\mathrm{WHO}$ and China CDC.

SARS-CoV-2 E gene subgenomic mRNA (sgmRNA), indicative of virus replication, was assessed by RT-PCR using an approach similar to previously described33, based on The primer and probe sequences (Forward : 5'-

GCTAGAGAACATCTAGACAAGAG-3', Reverse: 5'-ACACACGCATGACGACGTTATA-3', Probe : 5'-FAMTGTGATCGGTAGGAATGACGCGAAGC-Quencher-3').

Adoptive therapy of splenic T cells or immune sera in the mice challenged with live SARS-CoV-2

An adoptive therapy of splenic T cells was conducted previously24,36. hACE2 mice with C57BL/6 background received $5 \times 107$ 
splenic T cells isolated from either mice with same C57BL/6 background 9 days after the third dose of the candidate vaccine or from the mice treated with PBS as a control. The adoptive therapy of the sera was described previously27. The adoptive therapy based on immune sera was performed using $0.1 \mathrm{ml}$ of the pooled sera from the immunized mice at the same time. In addition, hACE2 mice with ICR background received $0.8 \mathrm{ml}$ sera from the mice 7 days after a single dose of the vaccine and challenged with live SARS-CoV-2. The mice were sacrificed 5 days after the challenge with live virus, and viral loads in lung tissues, lung histopathological changes, and body weight change were evaluated. Viral load in the lung tissues were measured by qRT-PCR. The primer sequences used were derived from the envelope (E) gene (Forward: 5'-TCGTTTCGGAAGAGACAGGT-3'; Reverse: 5'GCGCAGTAAGGATGGCTAGT-3'). Sections were stained by Hematoxylin and Eosin (H\&E) and evaluated under light microscopy.

Instrument

Software

Cell population abundance

Gating strategy
Flow cytometric data were collected using LSRFortessa (BD Biosciences) or NovoCyte (ACEA bioscience. Inc).

NovoExpress 1.4.1 or FlowJo v10.0.0.

We perform the flow cytometry for phenotypes of the memory T cells with the production of IFN-gamma or IL-4 by analysing the stimulated lymphocytes in the present study, and is easy to get enough CD8+ or CD4+cell for further gating memory cells.

Cells were gated from MHC-II- and CD45R-. 220-, CD4+ or CD8+ cells with CD44+ were defined as the memory cells reactive to $\mathrm{RBD}$ protein. From these gated cells the percentage of IL-4+ or IFN + + were recorded.

ACE2 transfected-Cells with FITC fluorescence intensity were counted for the inhibition of receptor binding assays.

Tick this box to confirm that a figure exemplifying the gating strategy is provided in the Supplementary Information. 